Bundesgesundheitsbl 2015 - 58:436-451

DOI 10.1007/s00103-015-2126-5

Online publiziert: 5. März 2015

(c) Springer-Verlag Berlin Heidelberg 2015
Claudia Ruscher · Martina Kraus-Haas • Alfred Nassauer · Martin Mielke

Fachgebiet für angewandte Infektions- und Krankenhaushygiene, Robert Koch-Institut, Berlin, Deutschland

\title{
Healthcare-associated infections and antimicrobial use in long term care facilities (HALT-2)
}

\author{
Deutsche Ergebnisse der zweiten \\ europäischen Prävalenzerhebung
}

\section{Zusatzmaterial online}

Die Online-Version dieses Artikels (doi: 10.1007/s00103-015-2126-5) enthält zusätzliches Material, das für autorisierte Benutzer zugänglich ist.

\section{Hintergrund}

Der demografische Wandel und damit die alternde Gesellschaft schaffen eine ständig wachsende Nachfrage nach Langzeitpflege und auch nach komplexer pflegerischer Versorgung in den Einrichtungen. Dies ist nicht allein dem Alterungsprozess der Gesellschaft geschuldet. Die Aufenthaltsdauer in Akutkrankenhäusern hat sich in den vergangenen Jahren stetig verkürzt und liegt aktuell bei durchschnittlich 7,6 Tagen [1]. Dies hat zur Folge, dass auch Patienten, die mitunter noch komplexer Pflege bedürfen, häufig früher in die Heimbetreuung zurück entlassen werden. Bei Eintritt in das Heim sind die Menschen im Durchschnitt 81 Jahre alt und verweilen dort 3,4 Jahre. In Deutschland sind ca. 2,9\% der Bevölkerung pflegebedürftig, das sind gegenwärtig 2,34 Millionen Menschen. Ein knappes Drittel davon $(31 \% ; n=717.490)$ wird stationär in Heimen versorgt. Der weit überwiegende Anteil der Pflegebedürftigen (95\%) in den Heimen ist über 60 Jahre alt und fast ein Viertel (20,5\%) ist schwerpflegebedürftig (Pflegestufe III). Vergleicht man diese Zahlen mit dem Anteil der Schwerpflegebedürftigen in der ambulanten Pflege $(11,6 \%)$ oder bei Menschen, die zu Hau- se durch Angehörige gepflegt werden $(7,7 \%)$, so erkennt man, dass der Anteil hier deutlich niedriger ist [2]. Die 12.354 stationären Pflegeheime in Deutschland sind also mit der Herausforderung konfrontiert, der Versorgung von Bewohnern, die immer älter werden und zudem häufiger komplexe Risikoprofile für Infektionen und Antibiotikabehandlungen aufweisen, gerecht zu werden.

Studien der vergangenen Jahre haben zudem gezeigt, dass Bewohner von Langzeitpflegeeinrichtungen mitunter eine bedeutende Rolle in der Epidemiologie von antibiotikaresistenten Bakterien wie MRSA, VRE oder ESBL-bildenden Bakterien spielen können. Fast immer handelt es sich bei den bei ihnen nachgewiesenen Erregern um die gleichen genetischen Linien, die auch bei Patienten in Krankenhäusern nachgewiesen werden [3-5].

Menschen im höheren Lebensalter haben aufgrund verschiedener Faktoren eine erhöhte Disposition gegenüber Infektionen. Hierzu zählt neben funktionellen Beeinträchtigungen (Schluckstörungen etc.) auch eine altersbedingte Dysfunktion der Immunantwort (Immunoseneszenz), die sowohl die unspezifische als auch die spezifische Immunität beeinträchtigt. Hinzu kommen chronische Grunderkrankungen, die eine Infektanfälligkeit begründen. Kommt es beim alten Menschen zu infektiösen Erkrankungen, so sind diese im Vergleich zu Infektionen bei jungen Menschen häufig schwieriger zu behandeln und mit erhöhter Letalität belastet. Spezifische Besonderheiten in der klinischen Manifestation (Fehlen von Fieber, atypische Manifestationen) erschweren zudem nicht selten die rasche klinische Diagnostik von Infekten.

Im Bereich der Langzeitpflege wird die individuelle Disposition gegenüber Infektionen ergänzt durch Faktoren der Exposition. Die Bewohner teilen die Versorgung mit Lebensmitteln, die räumliche Unterbringung sowie die pflegerische Versorgung, und Ausbrüche in den Einrichtungen sind häufig beschrieben [6]. Maßnahmen der Infektionsprävention werden limitiert durch den Anspruch, dass die soziale Betreuung der Bewohner und die langfristige Wahrung der Lebensqualität im Vordergrund stehen. Zudem führt die vorwiegend individuelle medizinische Versorgung der Bewohner durch die eigenen Hausärzte dazu, dass einrichtungsübergreifende Infektionspräventionsmaßnahmen bzw. die Umsetzung komplexer Konzepte und Strategien schwierig zu etablieren sind und ein regelmäßiger Austausch zwischen Pflegekräften und Haus- und Fachärzten oft nicht stattfindet. Dies betrifft insbesondere Aspekte der Infektionsprävention und der Antibiotikaverschreibung. Da es kaum einrichtungsweite Erfassungen dieser Zielgrößen gibt, ist für die Einrichtungen schwer erkennbar, ob es eine Häufung bestimmter Infektionsarten gibt oder wie viele Bewohner unter antibiotischer Therapie mit welchen Wirkstoffen stehen.

Im Jahr 2005 hat die Europäische Kommission empfohlen, dass die Mitgliedstaaten Surveillancesysteme zur Er- 
fassung des Antibiotikaeinsatzes in der Grund- und Regelversorgung etablieren bzw. weiter verstärken sollen [7]. Die kontinuierliche Erfassung und Bewertung von Infektionen und Erregern sowie die Erfassung des Antibiotikaverbrauchs sind in Deutschland mit der Änderung des Infektionsschutzgesetzes und weiterer Gesetze für klinische Einrichtungen gesetzlich verankert ( $\$ 23$ IfSG). Für den Bereich der stationären Pflege hingegen gibt es für dafür keine gesetzliche Grundlage, und so können Einblicke in diese Themen derzeit vor allem durch Inzidenz- bzw. Prävalenzstudien in ausgewählten Stichproben gewonnen werden.

In der Europäischen Union schätzt man die Zahl der Langzeitpflegeeinrichtungen auf ca. 62.000, mit einer Gesamtkapazität von etwa 3,1 Mio. Betten. Konservative Vorhersagen der Europäischen Kommission schätzen, dass sich der Bedarf nach Langzeitpflege in den nächsten 40 Jahren verdoppeln wird [14]. Um dieser Entwicklung auch auf Ebene der Infektionsprävention bei Menschen, die in Heimen gepflegt werden, Rechnung zu tragen, hat das ECDC das HALT-Projekt initiiert, das in Form von wiederholten Punktprävalenzerhebungen die Situation in den europäischen Pflegeeinrichtungen präzise abbilden soll.

\section{Das erste HALT-Projekt}

Im Jahr 2010 hat das Europäische Zentrum für die Prävention und Kontrolle von Infektionskrankheiten (ECDC) eine europäische Punktprävalenzerhebung zum Vorkommen von Infektionskrankheiten und zum Einsatz von Antibiotika in europäischen Pflegeheimen initiiert - das sogenannte HALT-Projekt (Healthcareassociated Infections in European Long Term Care Facilities). Insgesamt haben sich 28 europäische Staaten am HALTProjekt beteiligt. Im Zeitraum Mai bis September 2010 wurden dabei an einem von den Einrichtungen selbst gewählten Stichtag Daten von insgesamt 722 Heimen und 63.884 Heimbewohnern erfasst [8]. Damit wurde erstmals eine Punktprävalenzstudie nach einheitlichem Protokoll und einheitlichen Kriterien in einer Vielzahl von Einrichtungen in ganz Europa durchgeführt. Erfasst wurden dabei nicht nur die Prävalenz von Infektionen und systemischen Antibiotikabehandlungen, sondern auch relevante Strukturdaten der Einrichtungen (z. B. Ausstattungen mit Hygienefachkräften, Einzelzimmerausstattung, medizinische Versorgung der Bewohner), Risikofaktoren und Pflegecharakteristika der Bewohner sowie Inhalte von bestehenden Infektionspräventionskonzepten. In Deutschland haben sich an diesem ersten HALT-Projekt 73 Pflegeheime mit insgesamt 6496 Bewohnern beteiligt [9]. Die Beteiligung an der Studie war freiwillig und erfolgte mit Unterstützung durch die Gesundheitsämter. Die Erfassung erfolgte vor Ort durch die Pflegekräfte auf Basis eines einheitlichen Protokolls und standardisierter Fragebögen. Im Rahmen dieser Erhebung konnte gezeigt werden, dass in Deutschland in der Tat der weit überwiegende Anteil der Einrichtungen für die medizinische Versorgung der Bewohner die individuelle Hausarztbetreuung angab. Ein hohes Lebensalter, Inkontinenz, Desorientiertheit und Immobilität prägten in allen Ländern das Risikoprofil der Bewohner in den Einrichtungen. HALT hat eine relativ geringe Prävalenz an Infektionen $(0,79 \%)$ in Deutschland gezeigt; Harnwegsinfektionen, Haut-Weichgewebeinfektionen und Atemwegsinfekte waren dabei die am häufigsten dokumentierten Infektionen [9].

\section{HALT-2}

Obgleich HALT-1 nicht nur die Machbarkeit einer umfassenden europäischen Erhebung in Deutschland und anderen europäischen Ländern gezeigt hat, sondern auch erstmals wichtige Basisdaten für europäische Langzeitpflegeeinrichtungen generiert hat, konnten auch Aspekte identifiziert werden, die für eine höhere Qualität der gewonnenen Daten bei zukünftigen Erhebungen verändert oder ergänzt werden sollten. In Fortentwicklung der 2010 durchgeführten Erhebung wurde daher eine weitere Punktprävalenzerhebung nach einheitlichen, europäisch abgestimmten Methoden vom ECDC organisiert (HALT-2). Die Beteiligung an den HALT-Erhebungen beruht europaweit auf freiwilliger Teilnahme und kann so kaum Repräsentativität - weder national, noch auf internationaler Ebene - erreichen. Im
Zuge der zweiten Erhebung sollte daher sowohl die Zahl der teilnehmenden Länder als auch die Zahl der teilnehmenden Einrichtungen insgesamt nochmals deutlich erhöht werden. Zudem sollte mithilfe von verpflichtenden, intensiven Schulungsmaßnahmen für die beteiligten Einrichtungen sichergestellt werden, dass die Erfassung nach einheitlichen, standardisierten Methoden erfolgt und die Kriterien zur Erfassung sicher erkannt und verwendet werden.

Zur Erfassung von Infektionszeichen wurden im ersten HALT-Projekt Surveillancekriterien nach McGeer [10] und ärztliche Diagnosen zu Grunde gelegt. Diese Kriterien, die in den 1990iger Jahren speziell zur Infektionserfassung in Heimen erarbeitet wurden, wurden im Jahr 2012 von einer Arbeitsgruppe der amerikanischen Centers for Disease Control and Prevention (CDC) überarbeitet und evaluiert [11]. In HALT-2 wurden diese überarbeiteten Kriterien zur Erfassung der Infektionen zu Grunde gelegt, und die Erfassung der Infektionen sollte symptombasiert durch eigens geschultes Personal erfolgen. Zur weiteren Erhöhung der Datenqualität wurde zudem erstmals eine Validierung integriert, durch die in jedem Land die Qualität der gewonnenen Daten evaluiert werden kann.

\section{Methode}

\section{Rekrutierung der Einrichtungen}

Die Rekrutierung der teilnehmenden Einrichtungen erfolgte durch Einbindung der regionalen MRE-Netzwerke. Dafür wurden im Vorfeld Adressaten aus dem Öffentlichen Gesundheitsdienst und den regionalen Netzwerken aller deutschen Bundesländer angeschrieben und über die geplante Punktprävalenzerhebung informiert. Dem Anschreiben wurden Informationen in Form eines Flyers beigelegt, verbunden mit der Bitte, die Langzeitpflegeeinrichtungen im jeweiligen Zuständigkeitsbereich zu informieren sowie bei der Organisation und Koordination vor Ort unterstützend mitzuwirken. 
Bundesgesundheitsbl 2015 · 58:436-451 DOI 10.1007/s00103-015-2126-5

(c) Springer-Verlag Berlin Heidelberg 2015

\section{Ruscher · M. Kraus-Haas · A. Nassauer $\cdot$ M. Mielke}

\section{Healthcare-associated infections and antimicrobial use in long term care facilities (HALT-2). Deutsche Ergebnisse der zweiten europäischen Prävalenzerhebung}

\section{Zusammenfassung}

Infektionsprävention und Strategien zum angemessenen Einsatz von Antibiotika in Pflegeeinrichtungen haben vor dem Hintergrund des demografischen Wandels nicht nur in Deutschland eine große Bedeutung. Zur Schaffung einer verlässlichen Datenbasis und zur Identifikation relevanter infektionspräventiver Aspekte in diesem Bereich hat das European Centre for Disease Prevention and Control (ECDC) im Jahr 2013 die zweite Punktprävalenzerhebung zum Vorkommen von Infektionen und zum systemischen Antibiotikaeinsatz in europäischen Langzeitpflegeeinrichtungen initiiert (HALT-2). Neben methodischen Anpassungen zur Infektionserfassung wurde in dieser zweiten Erhebung das erfassende Personal im Vorfeld intensiv in der Erfassung und der Methodik geschult.
Aus Deutschland haben sich 221 Einrichtungen an dieser Erhebung beteiligt, sodass Daten von insgesamt 17.208 Bewohnern erfasst werden konnten. Gut etablierte Strukturen der regionalen MRE-Netzwerke haben die Rekrutierung der Teilnehmer sowie die Vorbereitung der Erhebung deutlich erleichtert. Die Medianprävalenz des systemischen Antibiotikaeinsatzes war mit 1,1\% (95\%-Cl 0,7-1,6) in den deutschen Heimen erneut vergleichsweise niedrig. Die am häufigsten eingesetzten Wirkstoffgruppen waren jedoch neben den Beta-Laktamen (Penicilline 18,2\%, andere Beta-Lactame $17,2 \%$ ) wieder vor allem Fluorchinolone (28,2\%). Die Erfassung der Infektionen erfolgte symptombasiert anhand von detaillierten Algorithmen entsprechend der kürzlich überarbeiteten McGeer-Surveillance- kriterien und ergab eine Medianprävalenz von 1,7\% (95\%-Cl 1,1-2,2). Symptomatische Harnwegsinfektionen (28,4\%), Haut- und Weichgewebeinfektionen $(27,9 \%)$ und Atemwegsinfektionen $(24,7 \%)$ wurden sowohl als häufigste Infektionsarten als auch als häufigste Indikationen für systemische Antibiotikabehandlungen identifiziert. Klinische Implikationen ergeben sich insbesondere durch den hohen Einsatz von Fluorchinolonen. Auf pflegerischer Seite besteht weiter im Bereich der Händehygienecompliance Verbesserungspotenzial.

\section{Schlüsselwörter}

Langzeitpflege · Antibiotikaeinsatz · Infektionsprävention · Alte Menschen . Prävalenz

\section{Healthcare-associated infections and antimicrobial use in long term care facilities (HALT-2). German results of the second European prevalence survey}

\section{Abstract}

Prevention of infections and strategies for the prudent use of antimicrobials in long-term care facilities have gained importance in view of the demographic changes, not only in Germany. To generate appropriate data and to identify relevant aspects of infection prevention in this field, the European Centre for Disease Prevention and Control (ECDC) launched the second point prevalence survey of healthcare-associated infections and antimicrobial use in European long-term care facilities in 2013 (HALT-2). Despite methodical adjustments in the collection of data on healthcare-associated infections, in this second survey healthcare workers in the participating facilities were intensively trained in methodology and data collection. Overall, 221 Ger- man facilities participated and collected data from 17,208 residents. Well-established structures of regional networks facilitated the recruitment of participants as well as the preparations for training and survey. The median prevalence of residents receiving at least one antimicrobial agent was 1.1\% (95\%-Cl 0,7$1,6)$ ), which is remarkably low. However, the most frequently used antimicrobials in German facilities beside beta-lactams (penicillins $18.2 \%$, other beta-lactams $17.2 \%$ ) were quinolones (28.2\%). Data collection of infections was performed based on signs and symptoms in detailed decision algorithms according to the recently updated McGeer surveillance criteria and yielded a median prevalence of $1.7 \%$ (95\%-Cl 1,1-2,2). Symptom- atic urinary tract infections (28.4\%), skin and soft tissue infection $(27.9 \%)$, and respiratory tract infections (24.7\%) were identified both as the most common types of infections and the most common indications for the use of systemic antimicrobials. Clinical implications evolve mainly from the high use of quinolones. In terms of infection prevention measures, compliance of health care workers with a hand hygiene regimen revealed further potential for improvement.

\section{Keywords}

Longterm care · Antibiotic use - Infection control · Elderly people $\cdot$ Prevalence

\section{Fortbildung der die Erhebung durchführenden Personen}

Zur Vorbereitung auf die Durchführung der Studie und die Fortbildung für die teilnehmenden Einrichtungen wurden zunächst alle nationalen Vertreter, durch das HALT-2 Management Team in einem zweitägigen Trainingskurs geschult, um Inhalte und Fortbildungsmaterialien der Fortbildungen in allen Teilnehmerländern einheitlich gestalten zu können und die Methodik gemeinsam zu diskutieren. In den folgenden beiden Monaten folgten eintägige Schulungen durch den nationalen Kontaktpunkt am RKI für Vertreter aller teilnehmenden Einrichtungen in sechs deutschen Städten. Dabei wurden die Teilnehmer in Form von Präsentationen über den Hintergrund, die Ziele und die Methodik von HALT-2 informiert sowie anhand von Fallbeispielen in der Datenerhebung geschult. Die Teilnahme an der Fortbildung war verpflichtend für die
Teilnahme an HALT-2, und die erforderlichen Studienunterlagen wurden den Teilnehmern erst bei der Fortbildungsveranstaltung ausgehändigt.

\section{Teilnahmekriterien}

\section{Einschlusskriterien}

In die Erhebung eingeschlossen wurden Einrichtungen, die ihren Bewohnern konstante Betreuung ( 24 von $24 \mathrm{~h}$ ) und qualifizierte Pflege bieten; also Anforderungen, 
die über die bloße Assistenz bei alltäglichen Erledigungen hinausgehen. Gleichzeitig sollten die Bewohner jedoch keine andauernde medizinische Spezialversorgung und keine invasiven medizinischen Maßnahmen benötigen. In die Erfassung integrierte Bewohner sollten in der Einrichtung leben, am Tag der Erhebung um $8 \mathrm{Uhr}$ morgens anwesend sein und seit mindestens $24 \mathrm{~h}$ in der Einrichtung aufgenommen sein, um ggf. auf eine vollständige Anamnese zurückgreifen zu können.

Die Einrichtungen sollten sich bei Teilnahme anhand verschiedener Kriterien $\mathrm{zu}$ einer der folgenden vom ECDC vorgegebenen Kategorien zuordnen, um eine bessere Vergleichbarkeit zu ermöglichen: Allgemeines Pflegeheim (Kategorie A), Altenheim/Seniorenresidenz (Kategorie B), Psychiatrische Einrichtung (Kategorie C), gemischte Einrichtung (Kategorie D) oder sonstige/andere Einrichtungen (Kategorie E).

\section{Ausschlusskriterien}

Ausgeschlossen wurden Langzeitpflegestationen in Krankenhäusern, Wohngruppen und -gemeinschaften, betreutes Wohnen, Obdachlosenunterkünfte sowie Tagespflegeeinrichtungen und ambulante Pflegedienste.

Bewohner, die nur Tagespflege beanspruchen oder sich am Erhebungstag stationär im Krankenhaus befinden, wurden nicht berücksichtigt.

\section{Instrumente der Erhebung}

Im Vorfeld des Projekts wurden erforderliche Datenschutz- und Ethikvoten eingeholt.

Die Erfassung der Daten basierte in jeder Einrichtung auf zwei verschiedenen Fragebögen. Im Einrichtungsfragebogen wurden Strukturdaten (z. B. Träger, Bettenzahl, Ausstattung mit Fachpersonal etc.), Nennerzahlen zu Pflegecharakteristika und Risikofaktoren aller Bewohner, Angaben zur medizinischen Versorgung und ihrer Koordination, zu Infektionskontrollmaßnahmen und zu Strategien zum Antibiotikaeinsatz erfasst. Die Einrichtungen wurden mithilfe einer CodeNummer aus datenschutzrechtlichen Gründen pseudonymisiert.
Der Bewohnerfragebogen wurde nur für Bewohner ausgefüllt, die am Tag der Erhebung eine systematische Antibiotikatherapie erhielten und/oder Beschwerden bzw. Symptome einer Infektion zeigten. Hier wurden Bewohnerdaten (z. B. Alter, Geschlecht, zurückliegende Krankenhausaufenthalte in den letzten drei Monaten) sowie Pflegecharakteristika und Risikofaktoren pseudonymisiert aufgenommen. Systemische Antibiotika und Antimykotika, Antituberkulotika sowie antibiotische Inhalationen wurden ebenfalls erfasst, lokale Antibiotika (z. B. im Rahmen von MRSA-Sanierungen) hingegen wurden nicht berücksichtigt. Außerdem konnten Ergebnisse mikrobiologischer Untersuchungen, incl. Resistenzdaten ausgewählter Erreger, in diesem Abschnitt angegeben werden. Infektionen wurden mithilfe von Algorithmen entsprechend den 2012 überarbeiteten Surveillancedefinitionen nach McGeer et al. erfasst [11]. Einzelne Beschwerden und Infektionssymptome konnten dafür in vorgegebenen Infektionsalgorithmen angekreuzt werden. Bei Erfüllung der erforderlichen Kriterien (z. B. bestimmte Anzahl/Kombination von Symptomen) führte der Algorithmus zu einer bestätigten Infektion. Andernfalls wurden nur einzelne Symptome ohne Infektionsbestätigung aufgenommen und nicht als Infektion gewertet. Bei Harnwegsinfektionen und tieferen Atemwegsinfektionen führte der Algorithmus ggf. auch zu der Option „wahrscheinliche Infektion“. Dies ist der Tatsache geschuldet, dass die Kriterien zur Bestätigung einer Harnwegsinfektion die Testergebnisse einer Urinkultur erfordern. Da dies in der Routine jedoch oft nicht geschieht, können mit der Option „wahrscheinliche Infektion“ vorhandene Symptome erfasst werden, auch wenn keine mikrobiologische Diagnostik vorliegt. Bei der Erfassung der Pneumonie ist laut Kriterien eine Röntgenthoraxaufnahme erforderlich, sodass auch hier die Option „wahrscheinliche Infektion“ der Erfassung von Symptomen ohne radiologische Diagnostik gegeben ist.

Infektionen wurden allerdings nur dann erfasst, wenn die Beschwerden bzw. Symptome am Erhebungstag vorhanden waren, oder wenn diese im Vorfeld vorhanden waren, der Bewohner am Erhe- bungstag aber noch aufgrund dieser Infektion antibiotisch behandelt wurde. In letzterem Fall wurde der zurückliegende Zeitraum auf max. 14 Tage begrenzt, um die zurückliegenden Infektionszeichen zuverlässig angeben zu können. Infektionen, die schon bei Aufnahme des Bewohners in die Einrichtung vorhanden oder in Inkubation waren, wurden nicht berücksichtigt. Das Ausfüllen des Bewohnerfragebogens erfolgte unter der Voraussetzung des Einverständnisses des jeweiligen Bewohners oder seiner gesetzlichen Vertreter.

\section{Erhebung}

Die Datenerhebung erfolgte in allen europäischen Einrichtungen an einem selbst gewählten Tag im Zeitraum zwischen dem 1. April und 31. Mai 2013. Sehr große Einrichtungen hatten auch die Möglichkeit, die Erhebung an zwei aufeinanderfolgenden Tagen durchzuführen.

\section{Validierung}

Die Datenvalidierung von HALT-2 basiert zum einen auf einer verblindeten Parallelerhebung und zum anderen auf schriftlichen Fallbeispielen, die während der Fortbildungsveranstaltungen durch die Teilnehmer zu bearbeiten waren. Der Validierung lag ein eigenes Protokoll zu Grunde. Für die Parallelerhebung sollten die nationalen Vertreter, die in der Surveillance erfahren sind, zeitgleich mit den lokalen Pflegekräften von zwei zufällig ausgewählten Einrichtungen die Erhebung verblindet durchführen. Dabei war gefordert, Daten von insgesamt mind. 100 Bewohnern zu erfassen. Die Ergebnisse dieser parallel durchgeführten Erfassung werden durch das HALT-2-Validierungsteam ausgewertet und sollen Einblicke in die Qualität der gewonnen Daten sowohl im Hinblick auf Strukturindikatoren der Infektionskontrolle und des Antibiotikamanagements in den Einrichtungen als auch im Hinblick auf Risikoprofile der Bewohner sowie Sensitivität und Spezifität der Daten zu Infektionen und Antibiosen liefern. Zudem soll mithilfe der Validierung die Übereinstimmung der in HALT-2 verwendeten überarbeiteten CDC-/McGeerKriterien aus dem Jahr 2012 und der al- 


\section{Originalien und Übersichten}

\begin{tabular}{|c|c|c|c|c|c|c|}
\hline & Alle & Kategorie $\mathrm{A}$ & Kategorie B & Kategorie C & Kategorie D & Kategorie E \\
\hline Anzahl der Einrichtungen & 220 & 126 & 41 & 2 & 48 & 3 \\
\hline \multicolumn{7}{|l|}{ Größe der Einrichtung } \\
\hline $\begin{array}{l}\text { Anzahl der in die Studie eingeschlos- } \\
\text { senen Bewohner }\end{array}$ & 17.208 & 8937 & 3841 & 76 & 3990 & 364 \\
\hline $\begin{array}{l}\text { Mittlere Größe der Einrichtungen (Bet- } \\
\text { tenzahl Median) }\end{array}$ & 80,5 & 77 & 92 & 41 & 80,5 & 132 \\
\hline $\begin{array}{l}\text { Größe der Einrichtung (Bettenzahl } \\
\text { Min-Max) }\end{array}$ & $15-316$ & 19-316 & $30-184$ & $30-52$ & $21-250$ & $15-239$ \\
\hline \multicolumn{7}{|l|}{ Träger (Anteil in \%) } \\
\hline öffentlich & 10,5 & 10,3 & 14,6 & 0,0 & 8,3 & 0,0 \\
\hline privat & 21,4 & 18,3 & 26,8 & 50,0 & 22,9 & 33,3 \\
\hline gemeinnützig & 67,3 & 69,8 & 58,5 & 50,0 & 68,8 & 66,7 \\
\hline keine Angabe & 0,9 & 1,6 & 0 & 0 & 0 & 0 \\
\hline \multicolumn{7}{|l|}{ Verfügbarkeit von qualifizierter Pflege } \\
\hline $\begin{array}{l}\text { Anteil der Einrichtungen, in denen } \\
\text { qualifizierte Pflege } 24 \text { von } 24 \text { Stunden } \\
\text { verfügbar ist (\%) }\end{array}$ & 97,3 & 97,6 & 97,6 & 100 & 95,8 & 100 \\
\hline \multicolumn{7}{|l|}{ Einzelzimmerausstattung } \\
\hline $\begin{array}{l}\text { mittlerer Einzelzimmeranteil in \% } \\
\text { (Median) }\end{array}$ & 79,4 & 77,8 & 83,3 & 84,9 & 73,5 & 87,9 \\
\hline Einzelzimmeranteil in \% (Min-Max) & $0,0-100$ & $8,3-100$ & $49,1-100$ & $76,9-92,9$ & $0,0-100$ & $30,0-100$ \\
\hline \multicolumn{7}{|c|}{ Die Daten für HALT-2 wurden erhoben von (Anteil in \%) ${ }^{1}$} \\
\hline ärztlichem Personal & 5,0 & 1,6 & 0 & 0 & 18,8 & 0 \\
\hline Pflegepersonal & 88,2 & 87,3 & 87,8 & 100 & 89,6 & 100 \\
\hline andere & 22,7 & 20,6 & 29,3 & 0 & 22,9 & 33,3 \\
\hline
\end{tabular}

ten, im ersten HALT-Projekt verwendeten Kriterien überprüft werden.

\section{Ergebnisse}

\section{Beteiligung und Strukturdaten der Einrichtungen}

Im Anschluss an die Rekrutierung durch die regionalen MRE-Netzwerke haben sich 339 Einrichtungen für das Projekt angemeldet und weitere Informationen angefordert. An den Fortbildungsveranstaltungen, die verbindlicher Bestandteil der Erhebung waren, haben 245 Einrichtungen mit jeweils einem oder mehreren Vertretern teilgenommen $(72,3 \%$ der Angemeldeten). Ausgefüllte Fragebögen sind im Erhebungszeitraum von 221 Einrichtungen zurück ans RKI gesandt worden (65,2\% der Angemeldeten; 90,2 \% der Fortgebildeten), eine Einrichtung wurde aufgrund unvollständiger bzw. inkonsistenter Datensätze im Nachgang aus der Analyse ausgeschlossen, sodass Daten von insgesamt 220 Einrichtungen in die europäische Erhebung eingegangen sind. Dies entspricht einem Anteil von 1,8\% der rund 12.400 nach SGB XI zugelassenen voll- bzw. teilstationären Pflegeheime in Deutschland [2].

Die meisten teilnehmenden Einrichtungen wurden in Baden-Württemberg (56; $25,3 \%)$, Nordrhein-Westfalen (54; $24,4 \%)$ und Hessen $(29 ; 13,1 \%)$ rekrutiert. Weitere Teilnehmer kamen aus dem Saarland (19; 8,6\%), Berlin (13; 5,9\%), Rheinland-Pfalz (10; 4,5\%), Hamburg (9; 4,1\%), Schleswig-Holstein (9; 4,1\%), Brandenburg (8; 3,6\%), Bayern (6; 2,7\%), Sachsen-Anhalt $(5 ; 2,3 \%)$, MecklenburgVorpommern $(2 ; 0,9 \%)$ und Niedersachsen $(1 ; 0,5 \%)$.

Die Erhebung wurde in $88,2 \%$ der Einrichtungen durch Pflegekräfte durchgeführt, 5\% der Einrichtungen gaben an, dass auch Ärzte an der Erhebung beteiligt waren, und in $22,7 \%$ waren auch andere Personen an der Erhebung beteiligt. So haben z. B. im Zuge von regionalen MRENetzwerkaktivitäten Doktoranden die Er- hebung in mehreren Einrichtungen eines Netzwerks durchgeführt (• Tab. 1).

Die weit überwiegende Zahl der Einrichtungen (95,5\%; 210 Einrichtungen) bietet ihren Bewohnern einen endgültigen Aufenthalt (bis zum Lebensende), 2,3\% (5 Einrichtungen) bieten einen langfristigen, aber nicht endgültigen Aufenthalt (mehr als 12 Monate) und 0,90\% (2 Einrichtungen) einen mittelfristigen Aufenthalt (3-12 Monate).

Relevante Strukturdaten der Einrichtungen (z. B. Größe, Träger, Einzelzimmeranteil, Verfügbarkeit von qualifizierter Pflege) sind getrennt nach Einrichtungskategorien in $\bullet$ Tab. 1 dargestellt.

\section{Medizinische Versorgung, Koordination und Ausstattung mit Hygienefachpersonal}

Die medizinische Versorgung der Bewohner wird in den Einrichtungen fast ausschließlich durch die Hausärzte der Bewohner gewährleistet. In 92,7 \% der Einrichtungen ist dies die alleinige Versor- 
gungsform, nur eine Einrichtung gab an, die Versorgung ausschließlich durch in der Einrichtung angestellte Ärzte zu gewährleisten (0,45\%). In 15 Einrichtungen $(6,8 \%)$ wird eine gemischte Versorgung durch Hausärzte und angestellte Ärzte angeboten. Im überwiegenden Anteil der Einrichtungen findet keine Koordination der medizinischen Maßnahmen auf Einrichtungsebene statt (62,3\%). Allerdings gaben 30,9\% der Einrichtungen an, medizinische Maßnahmen durch externe (also nicht in der Einrichtung angestellte) Ärzte zu koordinieren. Dies können z. B. bestimmte Hausärzte sein, die einen Großteil der Heimbewohner einer Einrichtung versorgen. Eine Koordination durch angestellte Ärzte erfolgt in 2,2\% der Einrichtungen, und in $4,6 \%$ erfolgt sie sowohl durch externe als auch durch intern angestellte Ärzte.

Das ECDC hat u. a. drei Kriterien bei der Einschätzung der Infektionspräventionsstrategien als Schlüsselkriterien hinterfragt: die Ausstattung mit Hygienefachpersonal ("infection control practitioner"), das Vorhandensein einer Hygienekommission sowie der Zugang zu externer Expertise in diesem Bereich (die Möglichkeit, sich externe infektionshygienische Beratung einholen zu können) [12].

Hygienefachpersonal (definiert als eine in der Infektionsprävention und -kontrolle geschulte Person) ist in 188 (85,5\%) der deutschen Einrichtungen verfügbar. Meist handelt es sich dabei um Pflegekräfte (in $82,9 \%$ der Einrichtungen), die als Hygienefachschwester/-pfleger entweder in der Einrichtung angestellt sind (66\%), extern die Einrichtung betreuen (8,33\%), oder es sind beide Varianten vorhanden $(25,6 \%)$. Ärzte üben nur in drei der Einrichtungen (1,6\%) diese Funktion aus. Eine gemischte Variante mit Ärzten und Pflegekräften wurde von 13,8\% der Einrichtungen angegeben.

Eine Hygienekommission ist in $64,1 \%$ der Einrichtungen $(n=141)$ etabliert, die Zahl der Sitzungen dieser Kommission pro Jahr reichen von 0 bis $>10$; die meisten Einrichtungen $(46,1 \%)$ gaben allerdings an, dass sich die Kommissionen zweimal jährlich treffen.

Zugang zu externer infektionshygienischer Beratung haben 187 Einrichtungen $(85 \%)$. Alle drei Schlüsselkriterien werden von 115 Einrichtungen erfüllt $(52,3 \%)$ während in 6 der Einrichtungen keiner dieser Parameter erfüllt ist (2,7\%).

\section{Händehygiene}

Fast alle Einrichtungen gaben an, dass sie im vergangenen Jahr Fortbildungen zur Händehygiene für das Personal durchgeführt haben (97,3\%). Diese Schulungen richteten sich in fast allen Einrichtungen $(98,13 \%)$ an die beschäftigten Pflegekräfte, in $25,7 \%$ der Einrichtungen auch an ärztliches Personal, in 25,7\% der Einrichtungen an das paramedizinische Personal (Physiotherapeuten, Logopäden etc.) und in $72,4 \%$ der Einrichtungen auch an das Reinigungspersonal.

Dem Personal steht in allen Einrichtungen alkoholisches Händedesinfektionsmittel zur Verfügung, alkoholische Tücher zur Händedesinfektion werden in $16,8 \%$ der Einrichtungen verwendet und in fast allen Einrichtungen (99,5\%) steht Flüssigseife zum Händewaschen zur Verfügung. Keine Einrichtung gab an, Stückseife zum Händewaschen bereit zu halten. Als häufigste Methode der Händehygiene gaben 97,3\% der Einrichtungen die Händedesinfektion mit einem alkoholischen Händedesinfektionsmittel an, eine Einrichtung $(0,4 \%)$ gab das Händewaschen mit antiseptischer Seife an, und fünf Einrichtungen machten hierzu keine Angaben $(2,3 \%)$.

Insgesamt 193 Einrichtungen (87,7\%) machten auch Angaben zum Verbrauch von alkoholischen Händedesinfektionsmitteln im zurückliegenden Jahr. Im Median wurden von den Einrichtungen demnach in 2012 7,13 ml Händedesinfektionsmittel pro Bewohnertag verbraucht, allerdings bei einer sehr großen Spannbreite (0,39-217,93 ml/Bewohnertag). Legt man die Menge von 3 ml Händedesinfektionsmittel pro durchgeführter Händedesinfektion zugrunde, entspricht dies im Median 2,4 Händedesinfektionen pro Bewohnertag (0,13-72,3).

\section{Infektionspräventionskon- zepte, Surveillance und Antibiotikastrategien}

Die in den Einrichtungen etablierten Infektionspräventionskonzepte und Stra- tegien sind in $\bullet$ Abb. 1 dargestellt. Am wenigsten etabliert sind Schulungen für ärztliches Personal (1,82\% der Einrichtungen bieten dies an) sowie Surveillancesysteme und die Rückkopplung der Ergebnisse dieser an das Personal (46,36\%). Schulungen für Pflegekräfte hingegen sind in fast allen Einrichtungen etabliert $(89,1 \%)$. Pflegestandards sind in $90 \%$ der Einrichtungen erarbeitet worden und fast alle Einrichtungen haben spezielle Maßnahmen zum Auftreten von multiresistenten Erregern bei Bewohnern festgelegt $(94,1 \%)$. Schriftliche Arbeitsanweisungen zum Umgang mit MRSA und zur Händehygiene sind in allen Einrichtungen verfügbar, Anweisungen zum Umgang mit Harnwegskathetern und PEGSonden bei fast allen (97,3\% und 96,8\%) und zum Umgang mit Gefäßkathetern in 44,1\% der Einrichtungen.

Ein Surveillancesystem zur Erfassung und Bewertung nosokomialer Infektionen ist in 12,7\% der Einrichtungen etabliert, während 83,6\% der Einrichtungen angaben, keine Surveillance durchzuführen, und 3,6\% keine Angaben dazu machten.

Antibiotikastrategien, die sich im europäischen Protokoll in erster Linie an Länder richten, in denen fest angestellte Ärzte in den Einrichtungen die Bewohner versorgen bzw. in denen auch Pflegekräfte und Krankenschwestern Antibiotika verordnen dürfen, sind in den deutschen Einrichtungen gar nicht $(79,1 \%)$ oder kaum etabliert. Hierzu zählen z. B. die Verfügbarkeit von jährlichen Antibiotika-Verbrauchsdaten oder lokalen Resistenzstatistiken sowie Antibiotika-Kommissionen und Therapierichtlinien inkl. Antibiotika-Listen. Eine Übersicht über die abgefragten Strategien und die Angaben der deutschen Einrichtungen gibt - Abb. 2.

\section{Bewohnerstruktur}

Als bedeutende Faktoren der Pflegeintensität haben sich auch im Rahmen dieser Erhebung wieder das hohe Lebensalter (> 85 Jahre; 51,3\%), räumliche und zeitliche Desorientiertheit (54,9\%), Inkontinenz (70,8\%) und Immobilität (Rollstuhlpflichtigkeit oder Bettlägerigkeit; 46,4\%) gezeigt. Abbildung 3 zeigt die Häufigkei- 


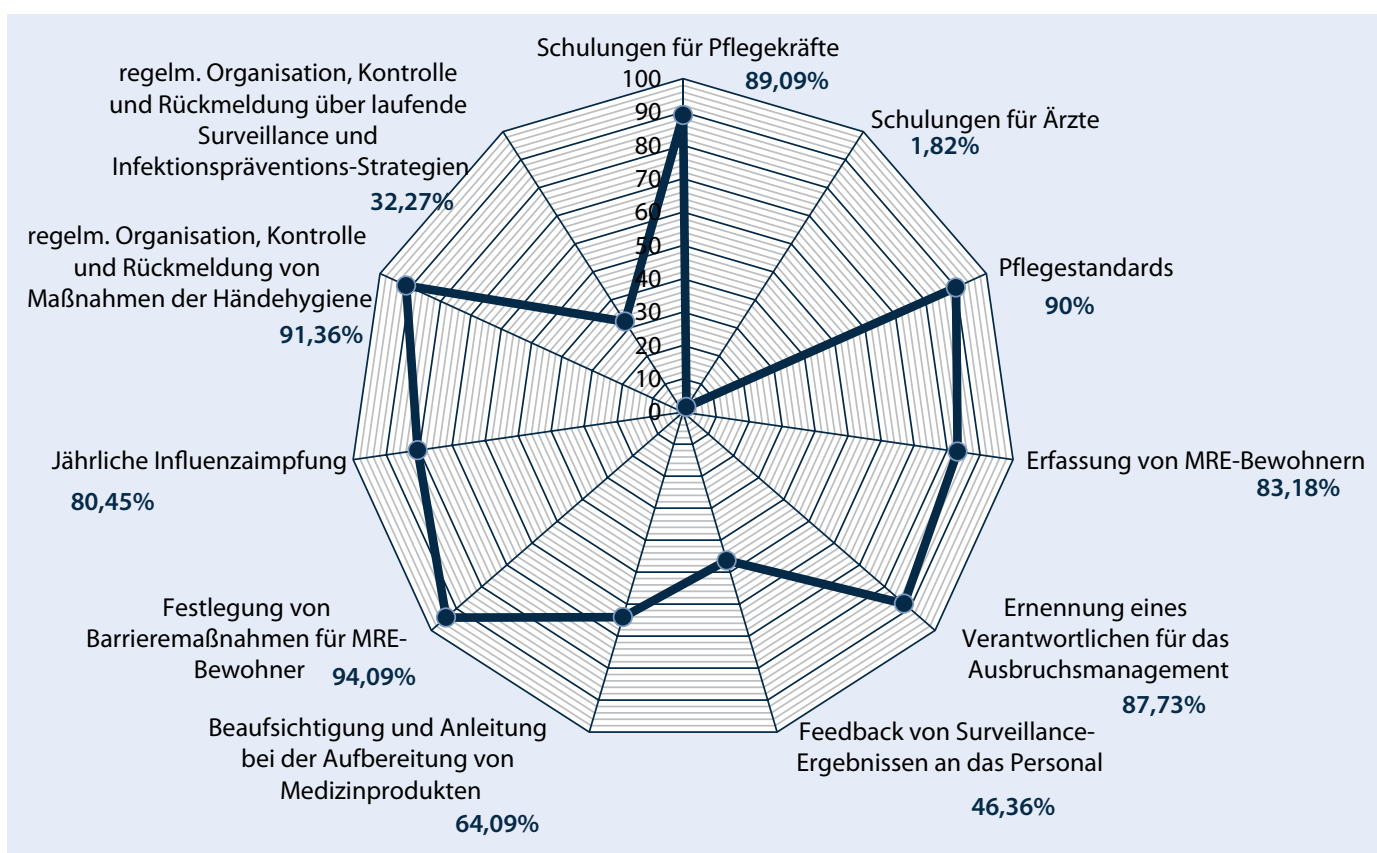

Abb. $1<$ Etablierte Infektionspräventionsstrategien in den Einrichtungen (Anteile der Einrichtungen, in denen die jeweilige Strategie implementiert ist, in Prozent): Ganz innen: 0\%; ganz außen: $100 \%$

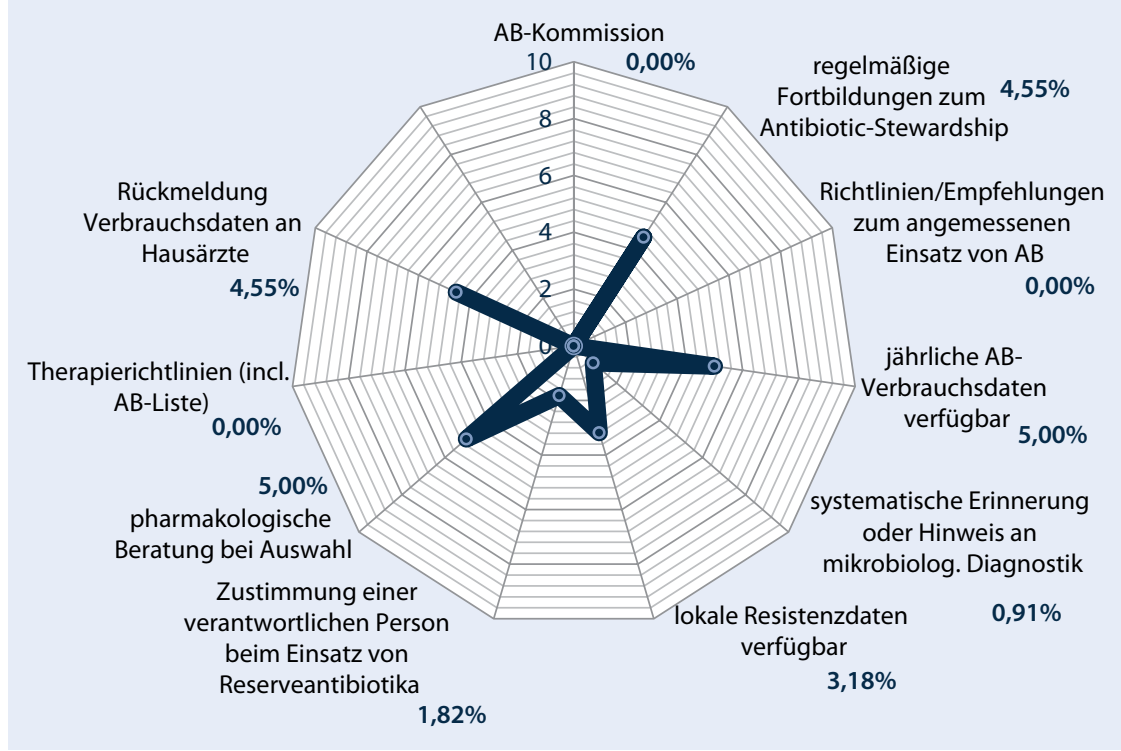

Abb. $2 \Delta$ Etablierte Antibiotika-Strategien in den Einrichtungen (Anteile der Einrichtungen, in denen die jeweilige Strategie implementiert ist, in Prozent)

ten der erhobenen Faktoren für die verschiedenen Einrichtungskategorien sowie in der Gesamtheit (jeweils Median \%).

\section{Infektionen}

Bei insgesamt 440 Bewohnern wurden Infektionen dokumentiert. Dies entspricht einer mittleren Prävalenz (Median) von $1,7 \%$ (95\% CI 1,1-2,2). Die rohe Prävalenz als Anteil von Bewohnern mit Infektionen unter allen Bewohnern lag bei
Atemwegsinfektionen $(24,7 \%)$ wurden am häufigsten dokumentiert (siehe - Abb. 4). Der weit überwiegende Teil der dokumentierten Harnwegsinfektionen $(90,1 \% ; n=118)$ entfiel hier jedoch in die Kategorie „wahrscheinliche Infektion", d. h. dass in aller Regel keine mikrobiologische Diagnostik durchgeführt wurde, die die Infektion bestätigt. Die Dokumentation beruhte in diesen Fällen allein auf Infektionszeichen und Symptomen anhand der Algorithmen. Die dokumentierten Atemwegsinfektionen waren hauptsächlich Infektionen der oberen Atemwege (Erkältung/Pharyngitis; $61 \%$, $n=70)$ sowie Infektionen der unteren Atemwege außer Pneumonien (29,2\%; $n=33)$. Die Gruppe der Haut-/Weichgewebeinfektionen wurde v. a. durch Haut-/ Bindegewebe-/Wundinfektionen repräsentiert $(84,4 \% ; n=108)$, gefolgt von Mykosen $(11,7 \% ; n=15)$ und Herpesinfektionen $(3,9 \% ; n=5)$.

Infektionen des Gastrointestinaltraktes repräsentierten $7,9 \%(n=36)$ der dokumentierten Infektionen, ein Viertel davon wurde durch Clostridium difficile verursacht $(n=9 ; 25 \%)$.

Die Verteilung der dokumentierten Infektionsarten und die genauere Zusammensetzung der häufigsten Arten sind in - Abb. 4 dargestellt. 


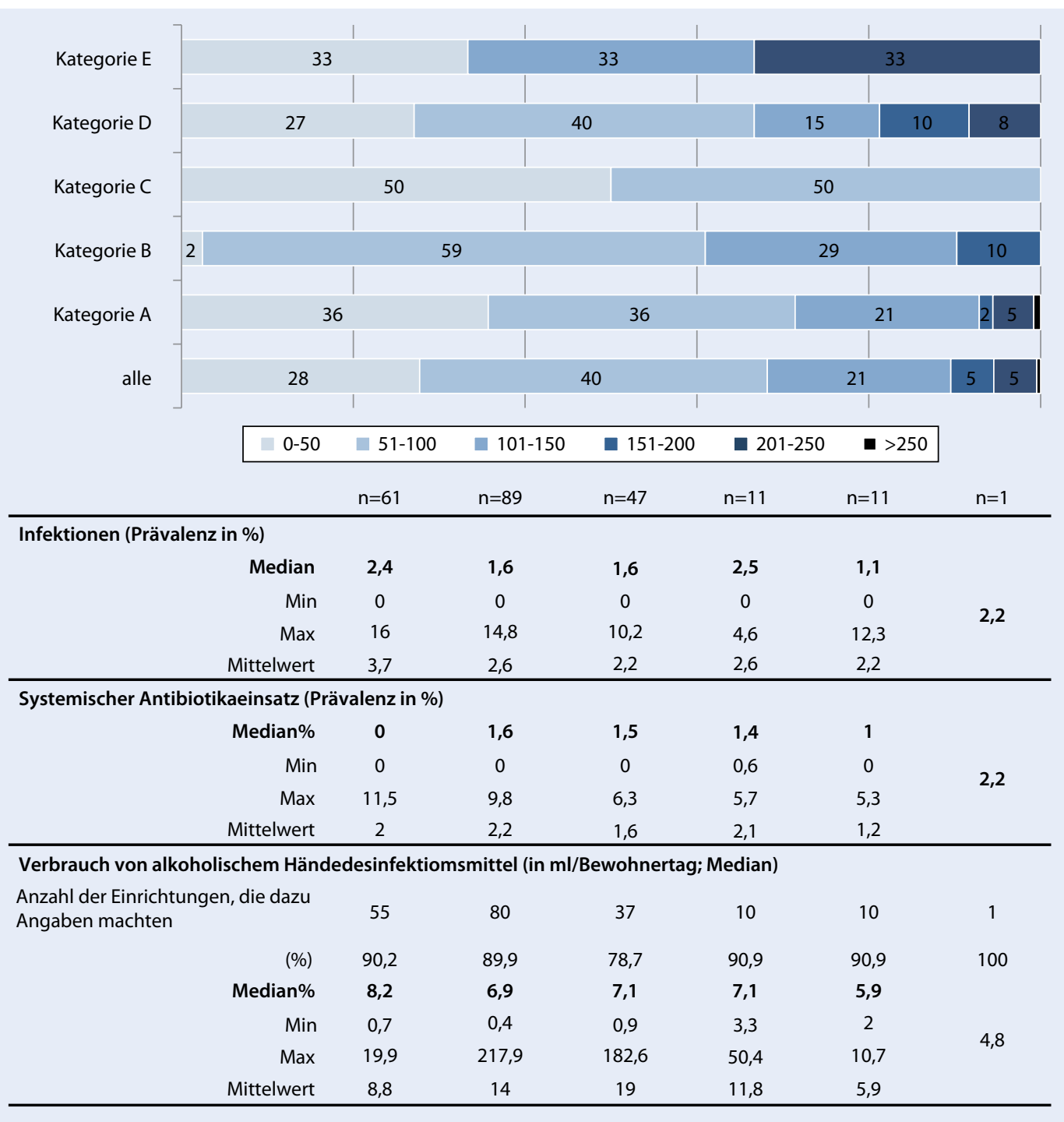

Abb. $3<$ Verteilung der teilnehmenden Einrichtungen nach Größe (Bettenzahl) in \% und Vergleich der Zielparameter nach Einrichtungsgröße

\section{Antibiotikaeinsatz}

Bei 324 Bewohnern wurde eine systemische antibiotische Behandlung dokumentiert. Dies entspricht einer Medianprävalenz von 1,1\% (95\%CI 0,7-1,6). Die rohe Prävalenz des systemischen Antibiotikaeinsatzes als Anteil von Bewohnern unter systemischer Antibiotikabehandlung von allen Bewohnern lag bei 1,9\%, war jedoch ebenfalls nicht normalverteilt (Min 0,0\%, P25 0,0 \%, P75 3,3\%, Max 11,5\%). Die Medianprävalenz in den Einrichtungskategorien ist in - Tab. $\mathbf{2}$ dargestellt. Insgesamt wurden 334 Antibiotika verordnet, zumeist in den Einrichtungen selbst $(78,4 \%)$. In Krankenhäusern wurden $11,1 \%$ der systemischen Antibiosen verordnet, $9,9 \%$ von anderer Stelle. Die behandelnden Hausärzte verordneten $71 \%$ der Antibiotika, 21,3\% wurden von Fachärzten und 2,8\% durch in den Heimen angestellte Heimärzte verordnet. Das geplante Enddatum aller antibiotischen Behandlungen war bei 75,9\% der Behandlungen bekannt bzw. in den Bewohnerakten vermerkt. Betrachtet man nur die therapeutischen Antibiosen, war dies häufiger der Fall $(81,8 \%)$, während bei prophylaktischen Antibiotikagaben nur bei 33,3\% das geplante Ende der Behandlung vermerkt war.

Die antibiotischen Behandlungen erfolgten fast ausschließlich oral $(97,6 \%)$, $0,6 \%$ wurden parenteral verabreicht und $1,8 \%$ auf anderen Wegen (z. B. inhalativ).

Die in den Einrichtungen am häufigsten eingesetzten antibiotischen Substanzklassen waren Fluorchinolone (J01M; $28,2 \%$ ) und Beta-Lactame (Penicilline
J01C; 18,2\%) sowie andere Beta-Lactame (Cephalosporine J01D; 17,2\%). Die am häufigsten eingesetzten Wirkstoffe insgesamt waren Ciprofloxacin (20,9\%), Cefuroxim $(11,4 \%)$, Nitrofurantoin $(8,1 \%)$ und Amoxicillin (7,1\%).

Antibiotika wurden am häufigsten verordnet für Infektionen der Harnwege $(44,9 \%)$ und der Atemwege (32,3\%). Haut- und Wundinfektionen waren die am dritthäufigsten dokumentierten Indikationen (12,6\%), gefolgt von Infektionen des Gastrointestinaltraktes $(4,2 \%)$ und "anderen Infektionen“ (2,1\%) (siehe - Abb. 5).

$12,6 \%$ der systemischen Antibiotikaeinsätze erfolgten prophylaktisch (um das Auftreten einer Infektion zu verhindern; d. h. der Bewohner zeigt zum Zeitpunkt der Verordnung keine Beschwerden oder 


\section{Originalien und Übersichten}

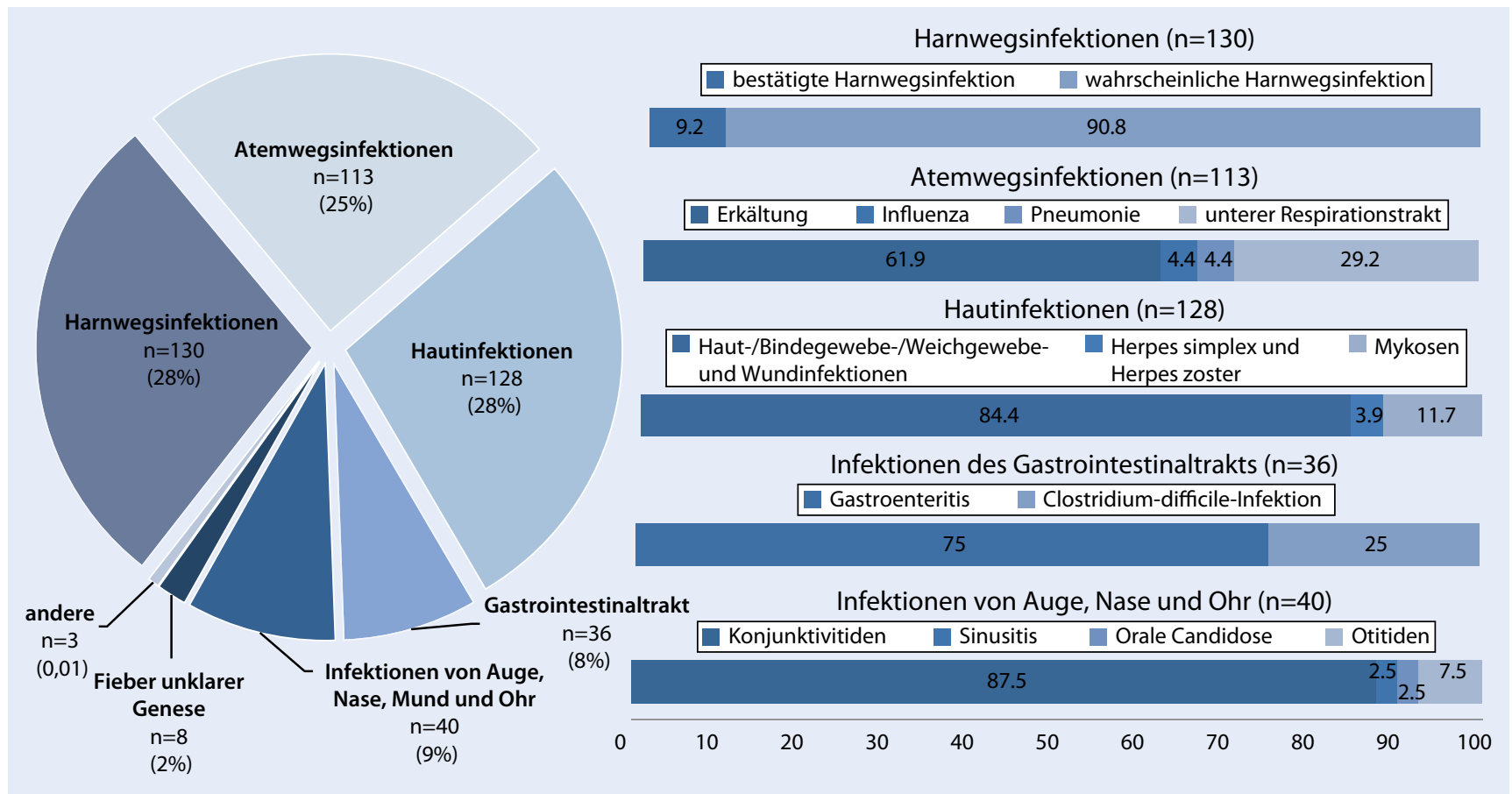

Abb. $4 \Delta$ Verteilung der Infektionsarten ( $n=458$ Infektionen bei 440 Bewohnern)

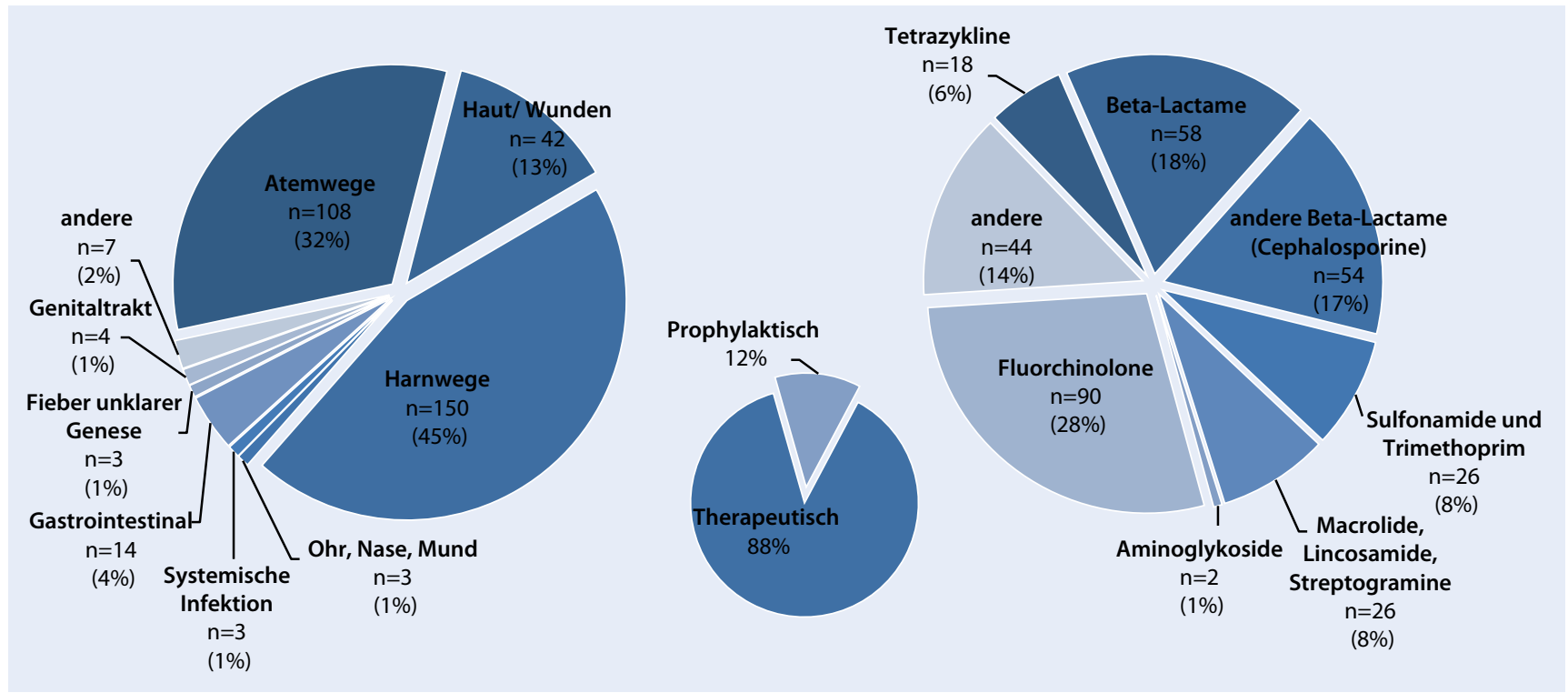

Abb. $5 \Delta$ Einsatz systemischer Antibiotika in den teilnehmenden Einrichtungen: Indikationen und Wirkstoffgruppen

Symptome einer Infektion). Die häufigste Indikation für die prophylaktische Antibiotikagabe waren die Harnwege (73,8\%) gefolgt von Haut und Wunden $(9,5 \%)$. Am häufigsten wurden Nitrofurantoine prophylaktisch verordnet $(n=21 ; 50 \%)$, zu gleichen Anteilen gefolgt von Cefuroxim, Cotrimoxazol und Ciprofloxacin (je $7,1 \%, n=3)$. Therapeutisch wurden in erster Linie die Harnwege (40,8\%) sowie die Atemwege (36,6\%) und Haut/Wunden $(13,0 \%)$ behandelt; am häufigsten mit Ciprofloxacin (22,9\%), Amoxicillin $(8,2 \%)$ sowie Doxycyclin $(5,8 \%)$, Nitrofurantoin (5,5\%) und Ampicillin-Sulbactam (Sultamicillin; 3,4\%).

Insgesamt wurden $41,5 \%(n=190)$ der dokumentierten Infektionen antibiotisch behandelt. Dieser Anteil ist bei den Harnwegsinfektionen mit 69,23\% am höchsten. Hier wurden 80 der 118 wahrscheinlichen Harnwegsinfektionen antibiotisch behandelt, das entspricht 67,8\%. Bei den bestätigten Harnwegsinfektionenist dieser Anteil nochmals deutlich höher: Von 12 bestätigten Harnwegsinfektionen wurden 10 antibiotisch behandelt (83,3\%). Der Anteil der antibiotisch behandelten Infektionen lag für die Atemwege bei 52,2\%. Innerhalb der Atem- 


\begin{tabular}{|c|c|c|c|c|c|c|}
\hline & Kategorie A & Kategorie B & Kategorie C & Kategorie D & Kategorie E & Alle \\
\hline & Allgemeines & Altenheim/ & Psychiatrische & Gemischte & andere & \\
\hline & Pflegeheim & Seniorenresidenz & Einrichtung & Einrichtung & & \\
\hline Anzahl Einrichtungen & 126 & 41 & 2 & 48 & 3 & 220 \\
\hline Anzahl Bewohner & 8937 & 3841 & 76 & 3990 & 364 & 17.208 \\
\hline $\begin{array}{l}\text { Anzahl der Bewohner mit } \\
\text { Infektionen }\end{array}$ & 218 & 84 & 1 & 109 & 28 & 440 \\
\hline $\begin{array}{l}\text { Median Prävalenz von Be- } \\
\text { wohnern mit Infektionen (\%) }\end{array}$ & 1,7 & 1,6 & 2 & 2,2 & 0 & 1,7 \\
\hline $\operatorname{Min}-\operatorname{Max}(\%)$ & $0,0-16,0$ & $0,0-14,8$ & $0,0-4,0$ & $0,0-15,0$ & $0,0-12,3$ & $0,0-16,0$ \\
\hline $\begin{array}{l}\text { Mittlere Anzahl von Infek- } \\
\text { tionen pro Bewohner mit } \\
\text { Infektion }\end{array}$ & 1,03 & 1,04 & 1 & 1,05 & 1,14 & 1,04 \\
\hline $\begin{array}{l}\text { Anzahl der Bewohner } \\
\text { unter Antibiose }\end{array}$ & 184 & 54 & 0 & 74 & 12 & 324 \\
\hline $\begin{array}{l}\text { Median Prävalenz von } \\
\text { Bewohnern unter Anti- } \\
\text { biose (\%) }\end{array}$ & 1,3 & 1,0 & 0,0 & 1,3 & 0,0 & 1,1 \\
\hline Min-Max (\%) & $0,0-11,5$ & $0,0-8,2$ & 0 & $0,0-10,5$ & $0,0-5,3$ & $0,0-11,5$ \\
\hline $\begin{array}{l}\text { Mittlere Anzahl von Anti- } \\
\text { biotika pro Bewohner unter } \\
\text { Antibiose }\end{array}$ & 1,0 & 1,1 & 0,0 & 1,1 & 1,0 & 1,0 \\
\hline
\end{tabular}

wegsinfekte war dieser Anteil am höchsten $(100 \%)$ bei den fünf bestätigten Pneumonien und bei $81,8 \%$ der 33 anderen unteren Atemwegsinfektionen. Aber auch zwei der fünf Influenza-Patienten (40\%) und $35,7 \%$ der Erkältungen (25 von 70 ) wurden antibiotisch therapiert. Infektionen von Haut- und Weichgewebe wurden in 23,5\% antibiotisch behandelt, am größten war der Anteil innerhalb dieser Gruppe mit 26,9\% bei den Wundinfektionen. Eine der 5 Herpesinfektionen (20\%) wurde ebenfalls antibiotisch behandelt; allerdings keine der 15 Mykosen. Infektionen des Gastrointestinaltraktes wurden zu 22,2\% antibiotisch behandelt. Den größten Anteil daran haben Infektionen durch Clostridium difficile, die in 5 von 9 Fällen antibiotisch behandelt wurden (55,6\%). Gastroenteritiden wurden in $11,1 \%$ antibiotisch therapiert.

Betrachtet man die Prävalenz der Risikofaktoren und Pflegecharakteristika vergleichend zwischen Bewohnern unter antibiotischer Behandlung und/oder Bewohnern mit Infektionen mit der Gesamtheit, zeigt sich, dass abgesehen vom Lebensalter alle Charakteristika bei Bewohnern mit antibiotischer Behandlung und/oder Infektionen häufiger vorkommen. Dies betrifft insbesondere zurückliegende Krankenhausaufenthalte, Ope- rationen, Harnwegskatheter, Immobilität sowie das Vorkommen von Wunden (- Abb.6a).

\section{Isolierte Mikroorgansimen und antimikrobielle Resistenz}

Die Erfassung der Daten zu Mikroorganismen und antimikrobieller Resistenz ist in HALT-2 gekoppelt an die Daten zur antibiotischen Therapie, das heißt, beides kann nur verbunden mit einer antibiotischen Behandlung im Fragebogen angegeben werden und nicht in Verbindung mit den Daten zu Infektionen. Dies bedeutet, dass im Falle einer Erregerisolierung, die im Zusammenhang mit der Infektionsdiagnostik und nicht zur Steuerung einer antibiotischen Behandlung erfolgte, diese Befundung nicht erfasst wird. Diese Methodik hat historische Gründe und ist seitens des ECDC bzw. der HALTKoordination so vorgesehen. Hier unterscheidet sich das HALT-2-Protokoll allerdings von dem der ECDC-Punktprävalenzerhebung (PPS) in Krankenhäusern, wo die Erfassung der Erreger an die Erfassung der Infektionen gebunden war. Dies muss bei der Auswertung und Interpretation der Erregerdaten berücksichtigt werden. Um HALT-2 dennoch in möglichst vielen Aspekten mit der ECDC-PPS in
Krankenhäusern zu harmonisieren und die Erfassung der Erregerresistenzen vor Ort zu vereinfachen, wurden Resistenzdaten nur von ausgewählten Wirkstoff-/ Bakterienkombinationen erfasst (z. B. 3.-Generation-Cephalosporin- und Carbapenemresistenz bei Enterobacteriaceae oder Methicillinresistenz bei S. aureus).

$84,7 \%$ der antibiotischen Verordnungen erfolgten empirisch, das heißt, es wurde vor Behandlungsbeginn keine mikrobiologische Kultur entnommen, um die Auswahl des geeigneten Antibiotikums zu stützen bzw. zu steuern. Für 51 antibiotische Verordnungen (15,3\%) wurde angegeben, dass Untersuchungsmaterial für eine mikrobiologische Diagnostik entnommen wurde, die Ergebnisse dieser Untersuchungen waren jedoch in $43,1 \%$ ( $n=22)$ für die die Erhebung durchführenden Personen nicht verfügbar. Insgesamt wurden 29 Mikroorganismen identifiziert, am häufigsten Staphylococcus aureus ( $n=7 ; 24,1 \%$ davon 6 MRSA), Escherichia coli ( $n=7 ; 24,1 \%$; davon einmal mit dokumentierter Resistenz gegenüber 3.-Generation-Cephalosporinen), Clostridium difficile ( $n=4 ; 13,8 \%)$, Klebsiella spp. $(n=3 ; 10,3 \%)$ und Proteus mirabilis $(n=2 ; 6,9 \%)$.

Vor systemischer Antibiose der Harnwege (therapeutisch und/oder prophylak- 


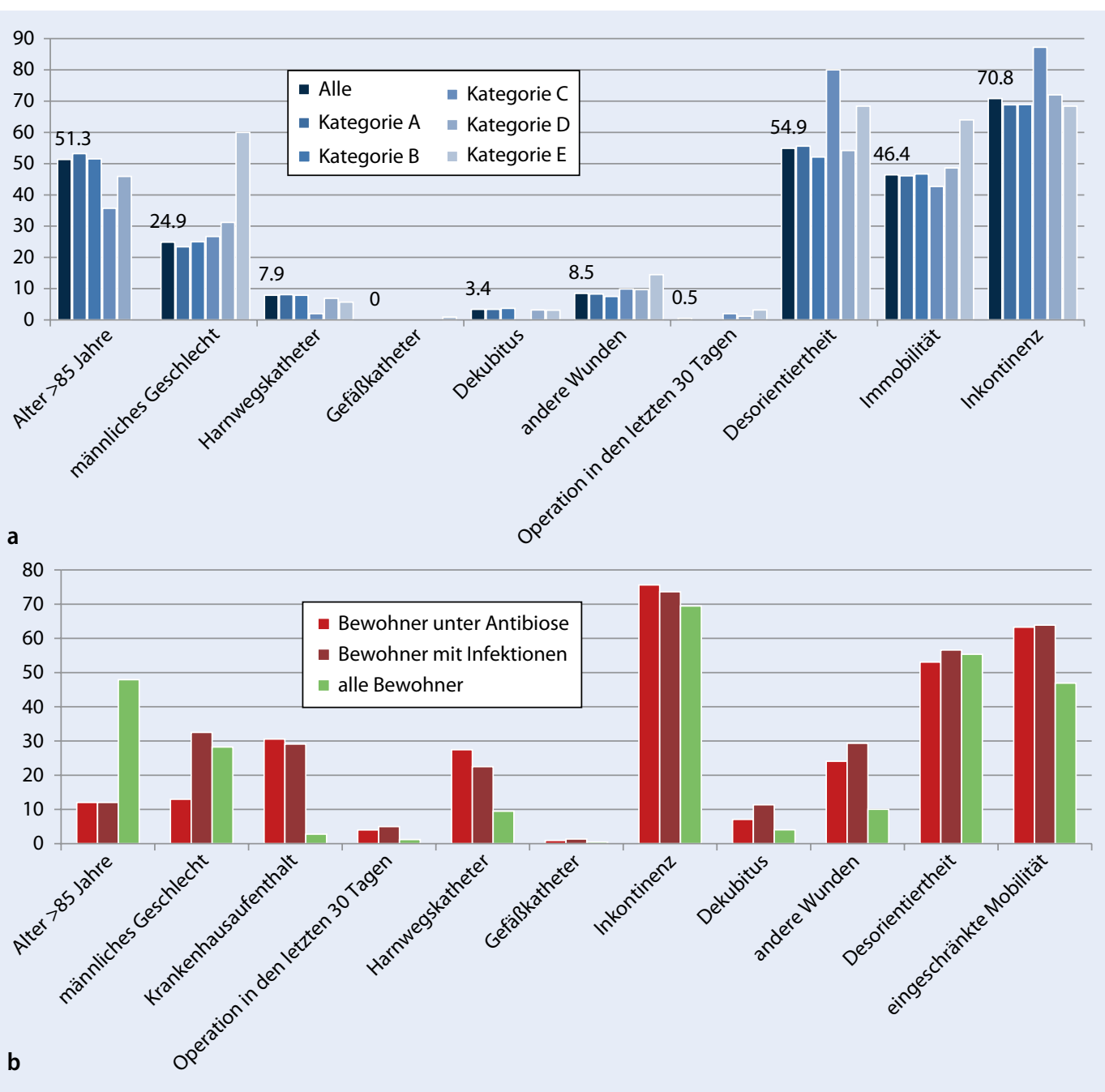

Abb. $6<$ a Prävalenz von Risikofaktoren und Pflegecharakteristika (\%) bei den Bewohnern der verschiedenen Einrichtungskategorien. b Prävalenz von Risikofaktoren und Pflegecharakteristika (\%) bei Bewohnern unter systemischer Antibiotikabehandlung, bei Bewohnern mit Infektionen und bei allen Bewohnern im Vergleich tisch) wurde in $20 \%$ der Verordnungen eine mikrobiologische Kultur entnommen $(n=30)$. In 16 dieser Untersuchungen $(53,3 \%)$ ist das Ergebnis der Einrichtung bzw. dem Pflegepersonal allerdings nicht zugänglich gewesen. Der häufigste aus den Harnwegen isolierte Erreger war Escherichia coli $(n=6)$. Aus den Atemwegen wurden vor Antibiotikaverordnung in 5 Fällen mikrobiologische Kulturen entnommen (4,6\%); in fast allen Fällen ist das Ergebnis dem Personal jedoch nicht zugänglich gewesen; aus einer Probe wurde $S$. aureus isoliert. Vor systemischer Antibiose von Haut- und Weichgeweben wurde insgesamt in 9,5\% mikrobiologische Proben entnommen $(n=4)$, aus denen $S$. aureus $(n=2), S$. epidermidis $(n=1)$ und in einem Fall eine Herpesvirusinfektion nachgewiesen wurde. Aus dem Gastrointestinaltrakt wurde in 28,6\% $(n=4)$ der systemischen Antibio- sen eine Kultur angelegt; in allen Fällen wurde Clostridium difficile als Erreger isoliert.

\section{Diskussion}

Mit der vorliegenden Punktprävalenzerhebung konnten umfangreiche Daten zum Vorkommen von Infektionen und zum Einsatz von Antibiotika sowie zu etablierten Infektionspräventionsstrategien in Deutschland und in europäischen Langzeitpflege-Einrichtungen erfasst werden. Auf der Basis des ersten HALTProjekts im Jahr 2010 (722 Einrichtungen aus 28 Ländern) wurden mit überarbeiteter Methodik nach einheitlichem Protokoll in 19 europäischen Ländern und insgesamt 1181 Einrichtungen die Prävalenz pflege- und therapieassoziierter Infektionen und des Antibiotikaeinsatzes sowie Strukturen der Infektionskontrol- le und ihrer Indikatoren erfasst, um eine zuverlässige Datenbasis zu schaffen und Potenzial für gezielte Präventionsmaßnahmen zu identifizieren [13]. Die Erhebung wurde dabei bewusst und in den europäischen Teilnehmerländern einheitlich in das Frühjahr gelegt, um insbesondere das Vorkommen endemischer Infektionen zu erfassen. Infektionsausbrüche z. B. durch Noro- oder Influenzaviren, wie sie vermehrt in den Wintermonaten auftreten, sollten nicht primärer Fokus der Erhebung sein.

Aus Deutschland haben sich an dieser zweiten Erhebung insgesamt 221 Einrichtungen beteiligt, was die Qualität gewonnen Daten gegenüber der ersten Erhebung in 2010 (mit 72 deutschen Einrichtungen) erhöht hat. Deutschland hat damit im europäischen Vergleich die zweithöchste Beteiligung nach Italien $(n=235$; Spanne international: 2-235 Einrichtun- 
gen/Teilnehmerland) [13]. Diese hohe Beteiligung wurde in erster Linie durch die enge Zusammenarbeit mit den in Deutschland inzwischen bundesweit etablierten regionalen MRE-Netzwerken ermöglicht, die vielerorts nicht nur die Information und Rekrutierung der Einrichtungen in ihrem jeweiligen Einzugsgebiet übernommen haben, sondern teils auch das eintägige Training selbst durchgeführt oder organisiert haben. Repräsentativität auf Ebene der Bundesländer bzw. auf Bundesebene wurde seitens des ECDC für das HALT-Projekt nicht gefordert, vielmehr stand es im Vordergrund, die Teilnehmerzahl insgesamt gegenüber der Erhebung in $2010 \mathrm{zu}$ erhöhen. Die sehr unterschiedlich hohe Beteiligung in den deutschen Bundesländern lässt jedoch erkennen, dass es in Bundesländern bzw. Regionen, die über eine inzwischen langjährige Netzwerkerfahrung verfügen und in denen die Strukturen regional entsprechend gut etabliert sind (z. B. Nordrhein-Westfalen, Hessen und Baden-Württemberg), leichter möglich war, Teilnehmer für die Erhebung zu rekrutieren, als in Bundesländern, in denen diese Strukturen noch nicht auf eine längere Erfahrung zurückblicken können. Da die Netzwerke häufig auch selbst mit ihren Moderatoren in den Schulungen präsent waren, waren sie den Einrichtungen bei Bedarf auch bei der Erhebung oder im Nachgang bei der Interpretation der gewonnen Daten behilflich. Die in Deutschland inzwischen bundesweit etablierten MRE-Netzwerke haben somit maßgeblich zum Erfolg des Projekts beigetragen bzw. seine Durchführung in dieser Breite überhaupt erst ermöglicht, was zeigt, dass die Netzwerkstrukturen sich nicht nur im praxisorientierten Umgang mit resistenten Erregern bewähren, sondern auch im Hinblick auf epidemiologische Erhebungen geeignete Strukturen darstellen.

Die Prävalenz des systemischen Antibiotikaeinsatzes ist in HALT-2 in den beteiligten deutschen Einrichtungen mit $1,1 \%$ nicht nur identisch mit der ersten Erhebung in 2010 (1,15\%), sondern auch zu einer Punktprävalenz-Erhebung, die im Jahr 2009 als Teil des European Surveillance of Antimicrobial Consumption (ESAC)-Nursing Home Projects in acht deutschen Langzeitpflegeeinrichtungen durchgeführt wurde $(1,18 \%)[14,15]$. Sie scheint also ein recht realistisches Abbild des Antibiotikaeinsatzes in deutschen Pflegeinrichtungen zu geben, was nicht zuletzt darauf zurückzuführen ist, dass die Erfassung dieser Daten immer auf der Dokumentation in den Bewohnerakten durch die betreuenden Hausärzte beruht. Sie unterliegt also - anders als die Erfassung der Daten zu Infektionen - kaum methodischen Änderungen und lässt sich so auch gut im Verlauf der HALT-Projekte und seiner Vorgänger vergleichen. Bei der Interpretation muss jedoch auch berücksichtigt werden, dass die teilnehmenden Einrichtungen sich an regionalen MRE-Netzwerken beteiligen und über diese rekrutiert wurden. Es ist also möglich, dass es sich um Einrichtungen handelt, die sich durch besonderes Engagement bzw. Sensibilisierung auf dem Gebiet des Antibiotikaeinsatzes und der Prävention multiresistenter Erreger auszeichnen, und dies die Daten beeinflusst.

Auch die europäischen Ergebnisse von HALT-2 zeigen mit einer mittleren Antibiotika-Prävalenz von $4,4 \%$ wieder fast identische Größen wie in der ersten Erhebung [12]. Die Gesamtprävalenz der systemischen Antibiotikagaben in den europäischen Teilnehmereinrichtungen lag in HALT-1 bei $4,3 \%$, und auch im Vergleich mit den Gesamtprävalenzen, die 2009 für das ESAC-Nursing Home Project in europäischen Heimen ermittelt wurden (6,33\% im April und 5\% im November 2009), erscheint die Prävalenz der deutschen Einrichtungen konstant niedrig $[15,16]$. In Finnland wurden die Daten aus drei wiederholten Punktprävalenzerhebungen in den Jahren 2009 und 2010, die ebenfalls teilweise im Rahmen des ESAC-Nursing Home Projects erhoben wurden, im vergangen Jahr veröffentlicht und zeigten mit einer mittleren Prävalenz von $12,6 \%$ (mit einer Spanne von 3,2 bis 33,3\%) einen deutlich höheren Antibiotikaeinsatz [17]. Auch Irland (10,2\%), Schottland (7,3\%) und Holland (3,5\%) haben ihre nationalen Antibiotikaprävalenzen der ersten HALT-Studie veröffentlicht und zeigen teils deutlich höhere Prävalenzen [18].

Die Beta-Lactame (Penicilline mit 18,2\% und Cephalosporine, Monobactame und Carbapeneme mit 17,2\%) mach- ten mit insgesamt 35,4\% (HALT-1: 33,8\%) den größten Anteil aus, gefolgt von Fluorchinolonen (28,2\%; HALT-1: 31,4\%). Hier zeigen sich im europäischen Vergleich durchaus Unterschiede. Bei Betrachtung der Gesamteuropäischen Daten aus HALT-2 und HALT-1 in 2010 zeigt sich, dass insbesondere die Chinolone in den deutschen Heimen weit häufiger eingesetzt werden, als dies im europäischen Durchschnitt geschieht (HALT-2: $16 \%$; HALT-1: 15,5\%). Dies spiegelt auch die Verteilung der am häufigsten eingesetzten Substanzen (unabhängig von der Wirkstoffgruppe) wider. Hier ist in Deutschland in HALT-2 Ciprofloxacin mit 20,9\% die am häufigsten eingesetzte Substanz, während z. B. in den beiden ESAC-Erhebungen in 2009 Ciprofloxacin in den europäischen Heimen deutlich seltener eingesetzt wird (April 2009: 6,8\%, November 2009: 5,8\%) [16]. Während in den aus skandinavischen Einrichtungen publizierten Prävalenzstudien zwar höhere Antibiotikaprävalenzen beschreiben werden, ist dort der Anteil an Chinolonen meist deutlich geringer. In der Regel werden dort Methenamine, Trimethoprim oder Nitrofurane zur Uroprophylaxe eingesetzt $[17,19,20]$. Ein ähnlich hoher Anteil an Fluorchinolonen (24\%) ist in 2010 aus der italienischen Prävalenzstudie im Rahmen von HALT in 92 Einrichtungen hervorgegangen [21]. Mögliche Ursachen für den hohen Chinolonanteil in den deutschen Heimen wurden bereits im Rahmen von HALT-1 diskutiert und lassen sich womöglich noch immer auf die Tatsache zurückführen, dass die medizinische Versorgung der Heimbewohner in Deutschland maßgeblich durch die individuellen Hausärzte erfolgt und eine einrichtungsübergreifende Surveillance über den Antibiotikaverbrauch nicht erfolgt, während in anderen europäischen Ländern häufiger fest in den Einrichtungen angestelltes ärztliches Personal die Versorgung der Bewohner betreut. Den einzelnen Hausärzten ist häufig nicht bekannt, in welchem Umfang ein Wirkstoff wie z. B. Ciprofloxacin in der jeweiligen Einrichtung verabreicht wird und in welchem Zusammenhang dies mit dem Auftreten resistenter Enterobacteriaceae oder auch von MRSA in der Einrichtung stehen kann. 
Auch der Anteil der Antibiotikaverordnungen, der durch eine mikrobiologische Untersuchung gesteuert wird, ist in den deutschen Einrichtungen mit 15,3\% vergleichsweise gering. Etwa $85 \%$ der systemischen Antibiotikabehandlungen in den deutschen Heimen werden empirisch verordnet. Die europäischen Daten aus HALT-2 (75\%), HALT-1 (69\%) und dem ESAC-Projekt $(54,4 \%)$ zeigen hier geringere Anteile empirisch verordneter Antibiosen. Die Indikation einer mikrobiologischen Probennahme stellt ein Thema für einrichtungsweite Konzepte dar. Obgleich die Zahlen zum Antibiotikaeinsatz in den deutschen Einrichtungen also vergleichsweise niedrig sind, lässt sich im Hinblick auf die Verordnung von Chinolonen und die Häufigkeit mikrobiologisch gesteuerter systemischer Antibiotikatherapien auf ärztlicher Seite noch Präventionspotenzial erkennen.

Die häufigsten Indikationen für eine systemische Antibiotikabehandlung sind in Deutschland wie im europäischen Kontext Harnwegsinfektionen, Atemwegsinfektionen und Infektionen von Haut/Weichgewebe. Dies hat sich neben den europäischen Erhebungen auch in skandinavischen Prävalenzstudien gezeigt $[17,20,22]$.

Der Anteil prophylaktisch verordneter Antibiotika ist in den deutschen Heimen mit $12,6 \%$ vergleichsweise gering. Hier zeigen die europäischen Erhebungen im Mittel konstant mehr als doppelt so hohe Anteile an antibiotischen Prophylaxen (ESAC 2009 28,8\%; HALT-1 2010 27,7\%; HALT-2 27,2). Gemeinsamkeiten aus allen europäischen Erhebungen bestehen darin, dass die Prophylaxe in erster Linie die Harnwege betrifft. Allerdings gaben die deutschen Einrichtungen immerhin 9,5\% der prophylaktischen Antibiosen mit der Indikation „Haut/Weichgewebe/Wunden" an, was im europäischen Vergleich recht häufig ist. So wurden in HALT-2 insgesamt nur 4,9\% der Prophylaxen mit der Indikation Haut und Wunden verordnet; in HALT-1 nur 3,8\%. Ob eine systemische Antibiose bei älteren Heimbewohnern tatsächlich die geeignete Methode zur Prophylaxe von HautWeichgewebe-/Wundinfektionen ist bzw. inwieweit und mit welchen Substanzen eine auch antibiotische Uroprophylaxe sinnvoll ist, bleibt kritisch zu hinterfragen und kann z. B. Bestandteil von Schulungsmaßnahmen sein. Es gibt international nur wenige qualitativ hochwertige Studien, die sich mit der Optimierung des Antibiotikaeinsatzes im Bereich der Langzeitpflege beschäftigen. Immerhin haben amerikanische Untersuchungen darauf hinweisen können, dass Schulungen für Ärzte, lokal erarbeitete Guidelines und Feedback zu Antibiotikaverbräuchen und lokalen Resistenzen an die verschreibenden Ärzte einen Einfluss auf die Häufigkeit von antibiotischen Verordnungen in der Langzeitpflege haben können [23]. In einer Studie konnte zudem der Anteil empirisch verordneter Antibiotika, und insbesondere der empirische Einsatz von Fluorchinolonen durch eine auf Antibiogrammen basierende Intervention gesenkt werden [24]. Eine große kanadische Studie in 630 Langzeitpflegeeinrichtungen konnte außerdem zeigen, dass längerfristige antibiotische Behandlungen bei älteren Heimbewohnern nicht nur häufig sind, sondern ihre Verordnung auch stärker durch die Präferenzen des verordnenden Arztes als durch die jeweiligen Patienten- bzw. Bewohnercharakteristika beeinflusst werden [25]. Dies unterstreicht das Potenzial, das in Form von gezielten Antibiotic-Stewardship-Interventionen, Schulungen und lokalen Resistenzdaten im Bereich der Langzeitpflege ausgeschöpft werden kann.

Die Daten von HALT-2 zu isolierten Erregern und Resistenzen müssen mit Vorsicht und Zurückhaltung interpretiert werden. Es ist dabei zum einen zu berücksichtigen, dass mikrobiologische Kulturen als Untermauerung der geeigneten antibiotischen Therapie eben nur selten entnommen werden $(15,3 \%)$ und die Untersuchungsergebnisse in einem sehr groBen Anteil der entnommenen Proben $(43,1 \%)$ für die Einrichtungen nicht verfügbar oder nicht auffindbar sind. Zudem muss berücksichtigt werden, dass eine gewisse Unterschätzung dieser Art von Daten und Befunden der Punktprävalenzerhebung inhärent ist, da sie an einem einzelnen Stichtag erfolgt und keine Möglichkeit der nachträglichen Befundeingabe besteht.

Die dokumentierten Erreger, mit Staphylococcus aureus und Escherichia coli als häufigsten Vertretern, entsprechen dennoch auch den europäischen Ergebnissen beider HALT-Studien sowie anderer Untersuchungen, die das Spektrum mikrobiologischer Befunde bei Heimbewohnern ausgewertet haben [26]. Allerdings ist neben den Enterobacteriaceae und Staphylokokken erneut Clostridium difficile häufig isoliert worden. Neben anderen Risikofaktoren, wie zurückliegenden Krankenhausaufenthalten, hat sich auch der orale Einsatz von verschiedenen antimikrobiellen Wirkstoffen, insbesondere den oralen Cephalosporinen, aber auch Fluorchinolonen und Clindamycin in Studien immer wieder als Risikofaktor für eine Clostridium-difficile-Infektionen gezeigt [27, 28], sodass sich auch vor diesem Hintergrund Fortbildungen und Schulungen im Antibiotic Stewardship für Hausärzte, die mit der Versorgung von Heimbewohnern betraut sind, als notwendig und sinnvoll abzeichnen.

Bei der Interpretation der Daten zum Vorkommen von Infektionen sind die methodischen Anpassungen im Rahmen von HALT-2 zu berücksichtigen. Während in HALT-1 zur Infektionserfassung modifizierte McGeer-Kriterien verwendet wurden, die neben der Erfassung der Infektionszeichen nach Aktenlage auch die in den Bewohnerakten dokumentierte Diagnose des behandelnden Arztes berücksichtigten, mussten im Rahmen von HALT-2 die erfassenden Personen standardisierte Zeichen und Symptome von Infektionen in Form von Algorithmen bei jedem Bewohner erfassen, und während der Datenanalyse im Nachgang wurden die jeweiligen Definitionen darauf angewandt und entschieden, ob eine Infektion als solche gezählt wird oder die erforderlichen Kriterien nicht erfüllt sind. Infektionen entsprechend den modifizierten MCGeer-Kriterien konnten in HALT-1 nur in $59,6 \%$ der Bewohner mit Infektionszeichen bestätigt werden. Dieser signifikante Verlust an „Fällen“ war vermutlich die Folge einer Untererfassung, die einerseits durch ungeübte bzw. unerfahrene Erfasser sowie andererseits durch mangelhafte Dokumentation von Infektionszeichen und Symptomen in den Akten der Bewohner verursacht wurde. Die im Rahmen von HALT-2 geforderte Anwendung von Definitionsalgorithmen erforderte es, nach al- 
len Symptomen und Infektionszeichen bei den Bewohnern aktiv zu suchen. Um das Personal mit eben diesen Algorithmen und der Erfassung von Symptomen besser vertraut zu machen und eine europaweit einheitliche Methodik besser zu gewährleisten, war das Training in HALT-2 ein wesentlicher Schwerpunkt und Voraussetzung zur Teilnahme an dem Projekt. Die verwendeten Algorithmen basieren dabei auf den durch Surveillanceexperten des CDC (Centers of Disease Prevention and Control der USA) im Jahr 2012 überarbeiteten MCGeer-Kriterien [10]. Lediglich kleinere Anpassungen wurden seitens des ECDC an diesen Kriterien vorgenommen, um u. a. dem begrenzteren Zugang zu mikrobiologischer Labordiagnostik in Europa im Vergleich zu amerikanischen Einrichtungen Rechnung zu tragen. Dies betrifft vor allem die Definition der Harnwegsinfekte, für die die amerikanischen Kriterien eine Infektionsbestätigung nur bei vorliegender mikrobiologischer Befundung erlauben. Da in vielen europäischen Ländern/Einrichtungen Urinkulturen zur Diagnostik von Harnwegsinfekten nicht routinemäßig entnommen werden, wurde in HALT-2 die zusätzliche Infektionsebene der „wahrscheinlichen“ Infektion ergänzt, die die Mikrobiologie nicht erfordert. Nach ähnlichem Prinzip wurde auch bei der Bestätigung der Pneumonie durch Röntgendiagnostik verfahren.

Möglicherweise ist die im Rahmen von HALT-2 ermittelte Infektionsprävalenz der deutschen Heimbewohner bereits aus diesen Gründen mit 1,7\% höher als im Jahr 2010 bei Anwendung der damaligen "strengen“ Surveillancekriterien nach McGeer et al. (HALT-1: 0,79\%). Die damalige Prävalenz von Bewohnern mit mindestens einem Infektionszeichen hingegen stimmt mit der aktuellen Prävalenz recht gut überein (1,6\%). Ähnliches gilt damit auch für die Gesamtprävalenz der HALT-2-Teilnehmer in Europa (3,4\% in HALT-2 im Vergleich zu 2,4\% nach McGeer in HALT-1 und $4 \%$ mit mindestens einem Infektionszeichen). Im europäischen Vergleich ist die Infektionsprävalenz der deutschen Einrichtungen auch in HALT-2 niedrig.

Die methodische Anpassung im Rahmen von HALT-2 erklärt auch, dass der Anteil der Infektionen, die antibiotisch behandelt wurden, in HALT-1 deutlich höher war als in HALT-2 (67,3\% in HALT-1 und 41,5\% in HALT-2). Die Dokumentation von systemischen Antibiotikabehandlungen ist in den Bewohnerakten vermutlich häufiger als die einzelner Infektionszeichen oder Symptome ohne antibiotische Verordnung. Bei Erfassung nach Aktenlage werden daher vermutlich auch Infektionszeichen häufiger in Verbindung mit der verordneten Antibiose dokumentiert, was in HALT-1 zu einem scheinbar höheren Anteil antibiotisch therapierter Infektionen führte. Die symptombasierte Erfassung in HALT-2 hingegen hat auch nicht dokumentierte Infektionszeichen und solche, die ohne einhergehende Antibiotikabehandlung auftreten, ermittelt.

Atemwegsinfektionen, Harnwegsinfektionen und die Infektionen von Hautund Weichgewebe sind die häufigsten Infektionsarten bei Heimbewohnern. Die Anpassung der Surveillancekriterien für Harnwegsinfektionen hat sich für die Erfassung in den deutschen Heimen als sinnvoll erwiesen, da der weit überwiegende Teil der dokumentierten Harnwegsinfektionen allein auf Symptomen basiert und ohne mikrobiologische Bestätigung erfolgte.

In Frankreich wurde in den Jahren 2006 und 2007 eine große Periodenprävalenzerhebung mit fünf Erhebungen zum Vorkommen von Infektionen durchgeführt, die Daten von insgesamt $10 \%$ aller Heimbewohner Frankreichs enthielt. Auch hier wurden die McGeer-Kriterien mit entsprechenden Anpassungen an mikrobiologische (Harnwegsinfektionen) und radiologische (tiefe Atemwegsinfektionen) Diagnostik zu Grunde gelegt. Von insgesamt $11,23 \%$ der Bewohner mit Infektionszeichen ergab sich bei Anwendung der adaptierten McGeer-Kriterien eine Prävalenz von 6,63\%. Die symptomatischen Harnwegsinfektionen, Infektionen der Atemwege sowie der Haut-/ Weichgewebe machten auch in dieser sehr großen Erhebung die häufigsten Infektionsarten aus [29].

Im Rahmen von HALT-2 wurden erstmals auch detaillierte Informationen zum Händehygienemanagement in den Einrichtungen erhoben. Alle Einrichtungen gaben an, dass ihnen alkoholisches
Händedesinfektionsmittel zur Verfügung steht, und fast alle haben die hygienische Händedesinfektion auch als die wichtigste Maßnahme der Händehygiene in ihrer Einrichtung benannt. International gibt es nur wenig empirische Studien zur Händehygiene in Pflegeheimen, und die existierenden sind aufgrund sehr geringer Stichprobengrößen nur schwer zu vergleichen bzw. ihre Erkenntnisse zu verallgemeinern [30]. Im Hinblick auf das inzwischen endemische Vorkommen resistenter Erreger wie MRSA oder 3.-Generation-Cephalosporin-resistenter Enterobakterien in pflegerischen Einrichtungen kommt der Händehygiene jedoch gerade dort eine besonders große Bedeutung zu. Die hygienische Händedesinfektion ist seitens des CDC und der WHO zur wichtigsten Standardmaßnahme der Händehygiene in Einrichtungen des Gesundheitswesens erklärt worden und kann bei korrekter Durchführung zu einer drastischen Senkung der Zahlen nosokomialer Infektionen führen $[31,32]$. Auch für den Pflegesektor konnte dies bereits gezeigt werden. In einer amerikanischen Interventionsstudie wurde der Einfluss einer Händehygieneintervention mit dem Schwerpunkt auf alkoholischer Händedesinfektion von Personal und Bewohnern auf die Infektionsraten in einem großen Pflegeheim untersucht und eine signifikante Senkung der Infektionsraten des unteren Respirationstraktes gezeigt [33].

Im Rahmen von HALT-2 machten $87,7 \%$ der deutschen Einrichtungen $(n=193)$ auch Angaben zur Verbrauchsmenge alkoholischer Händedesinfektionsmittel in ihrer Einrichtung im zurückliegenden Jahr (2012). Im Median hat sich hieraus eine Verbrauchsmenge von 7,49,8 ml/Bewohnertag (je nach Kategorie) ergeben. Obgleich diese Angaben mit einer großen Spannbreite und einer entsprechenden Ungenauigkeit behaftet sind, zeigen sie nicht nur die Machbarkeit der Erfassung dieses Indikators, sie zeigen auch eine bemerkenswerte Übereinstimmung mit den im Rahmen des Surveillancesystems KISS erhobenen Verbrauchsmengen in deutschen Pflegeeinrichtungen von im Median 9 ml/Bewohnertag (2013; 82 Einrichtungen). Legt man für eine durchgeführte Händedesinfektion eine Verbrauchsmenge von $3 \mathrm{ml}$ Händedesinfek- 
tionsmittel zu Grunde, entspricht dies 3 durchgeführten Händedesinfektionen pro Bewohnertag [34]. Berücksichtigt man zudem, dass fast alle deutschen HALT-2 Teilnehmer angaben, die Händedesinfektion mit einem alkoholischen Händedesinfektionsmittel sei in ihrer Einrichtung die wichtigste Maßnahme der Händehygiene, scheint sich dies aktuell noch nicht in den Verbrauchsmengen wider zu spiegeln. Legt man die von der „Aktion saubere Hände" [35] adaptierten Indikationen zur Händedesinfektion für den Bereich der Pflegeheime zugrunde (vor und nach medizinisch-pflegerischem Bewohnerkontakt, vor aseptischen Tätigkeiten, nach Kontakt mit potenziell infektiösem Material und nach Kontakt mit der direkten Bewohnerumgebung), wird deutlich erkennbar, wie viel Potenzial im Bereich der Händedesinfektion gegenwärtig noch ausschöpfbar ist. Eine finnische Interventionsstudie konnte beispielsweise mithilfe eines multidisziplinären Schulungsteams sowie regionalen Guidelines zum Antibiotikaeinsatz sowohl den Verbrauch an alkoholischem Händedesinfektionsmittel signifikant erhöhen als auch den Einsatz antimikrobieller Wirkstoffe in Pflegeinrichtungen senken [36].

Relevante Unterschiede zwischen der Art der Träger oder der Größe der Einrichtungen haben sich im Hinblick auf die Prävalenzen von Infektionen und Antibiotikabehandlung oder die Infektionspräventionskonzepte in HALT-2 nicht ergeben. Obgleich sich in einzelnen Aspekten Unterschiede erkennen lassen (siehe elektronisches Zusatzmaterial Anhang 1 und 2), ist ein eindeutiger Trend, der das Ziehen von Rückschlüssen erlauben würde, nicht zu erkennen. Dies kann aber auch auf die recht ungleich repräsentierten Anteile zurückzuführen sein. So ist der weit überwiegende Anteil der Einrichtungen, die an HALT-2 teilgenommen haben, freigemeinnütziger Natur, während private und insbesondere öffentliche Träger zu kleineren Teilen vertreten sind. Eine kanadische Metaanalyse hat im Jahr 2009 zeigen können, dass sich auch die Trägerschaft von Pflegeeinrichtungen auf die Qualität der Pflege auswirkt. Dieser Auswertung zu Folge bieten öffentliche/freigemeinnützige Träger im Durchschnitt eine höhere Pflegequalität als pri- vate Träger [37]. Die große französische Studie hingegen, die in Form von Periodenprävalenzen $10 \%$ der französischen Heimbewohner einschloss, konnte keine Unterschiede in der Infektionsprävalenz zwischen den verschiedenen Trägern feststellen.

Die zweite europäische HALT-Erhebung konnte zusammenfassend im vergangenen Jahr mit einer deutlich erhöhten Teilnehmerzahl (sowohl deutschlandweit als auch in Europa) wichtige Daten zur Prävalenz von Infektionen und dem systemischen Einsatz von Antibiotika wie auch zu etablierten Konzepten und Strategien der Infektionsprävention in den Einrichtungen erfassen. Als relevante Themen im Hinblick auf interventionelle Maßnahmen und Schulungen haben sich auf ärztlicher Seite dabei insbesondere der Einsatz von Fluorchinolonen zur Therapie und Prophylaxe von Infektionen bei Heimbewohnern sowie die systemische antibiotische Therapie von Haut-/ Weichgewebeinfektionen abgezeichnet, während dies auf pflegerischer Seite insbesondere der Bereich der Händehygiene ist. Hier kann neben den meist ohnehin jährlich stattfindenden Fortbildungen auch eine Teilnahme an der nationalen Kampagne „Aktion saubere Hände" für die Einrichtungen hilfreich sein, da dort in Form eines eigens für den Pflegebereich erarbeiteten Moduls umfangreiches Material und Anleitungen zur Erfassung der Händehygienecompliance zur Verfügung gestellt werden.

Die Erhebung hat den Teilnehmern zudem insbesondere durch die intensive Schulung und das zur Verfügung gestellte Schulungsmaterial nicht nur die Möglichkeit gegeben, sich mit dem Thema der Infektionssurveillance und der Methodik vertraut zu machen und sich darin zu üben, sondern auch ein verstärktes Bewusstsein für diese Thematik in den Einrichtungen bewirkt. Die Implementierung (einfacherer und ggf. angepasster) Erfassungsinstrumente kann es den Einrichtungen vor Ort ermöglichen, Entwicklungen im Bereich pflege-und therapieassoziierter Infektionen sowie im Bereich des Antibiotikaeinsatzes individuell und unabhängig zu verfolgen und prioritäre Maßnahmen für lokale Interventionen selbst zu erkennen. Obgleich aufgrund der Freiwilligkeit der Teilnahme und der Rekrutierung über die regionalen Netzwerke keine Repräsentativität der Daten für Deutschland gewährleistet ist, hat die Kategorisierung der teilnehmenden Einrichtungen es den HALT-2 Teilnehmern dennoch ermöglicht, sich gezielt mit Einrichtungen ähnlicher Struktur zu vergleichen. Von übergeordneter Bedeutung hat sich im Zuge der Erhebung die Einbindung der regionalen MRE-Netzwerke besonders im Hinblick auf die Rekrutierung und Information der Einrichtungen erwiesen. Dies kann und wird sicher auch bei der Implementierung von Erfassungsinstrumenten in einzelnen Einrichtungen oder der Umsetzung von festgestellten Fortbildungsbedarfen und Interventionen erfolgen. Auch der Austausch der Einrichtungen gleicher und verschiedener Kategorien im Hinblick auf die Ergebnisse von HALT und die sich daraus ergebenden Konsequenzen sowie zukünftige netzwerkinterne, regionale oder bundesweite Erfassungen können sich diese gut etablierten Strukturen zu Nutze machen.

\section{Korrespondenzadresse}

\section{Dr. C. Ruscher}

Fachgebiet für angewandte Infektionsund Krankenhaushygiene

Robert Koch-Institut

Nordufer 20, 13353 Berlin

ruscherc@rki.de

Danksagung. Wir möchten uns besonders bei allen Teilnehmern sowie den regionalen Netzwerken und Ansprechpartnern aus dem öffentlichen Gesundheitsdienst für die geleistete Arbeit und das große Engagement bedanken. Dem ECDC und der HALT-Koordinationsgruppe um Katrien Latour und Béatrice Jans gilt ebenso besonderer Dank für die Organisation der Studie und die Hilfestellung vor, während und nach der Erfassung. Frau Dr. Wischnewski hat durch große Unterstützung in der Planung der Studie und der Fortbildungen sowie bei der Validierung einen besonderen Beitrag geleistet, für den wir uns herzlich bedanken möchten. Aus dem Fachgebiet 14 des Robert Koch-Instituts möchten wir uns bei Frances Wiesner für die herausragende organisatorische Unterstützung sowie bei Katja Levin und Manuela Hanisch für die große Hilfe bei der praktischen Umsetzung bedanken.

\section{Einhaltung ethischer Richtlinien}

Interessenkonflikt. C. Ruscher, M. Kraus-Haas, A. Nassauer und M. Mielke geben an, dass keine Interessenkonflikte bestehen 


\section{Literatur}

1. Destatis Statistisches Bundesamt (2012) Krankenhäuser: Einrichtungen, Betten und Patientenbewegung. https://www.destatis.de/DE/ZahlenFakten/ GesellschaftStaat/Gesundheit/Krankenhaeuser/Tabellen/KrankenhaeuserJahre.html. Zugegriffen: 1. Okt 2014

2. Statistisches Bundesamt, Pfaff H (2011) Pflegestatistik. Pflege im Rahmen der Pflegeversicherung Deutschlandergebnisse - 2009. Statistisches Bundesamt, Wiesbaden

3. Ruscher C, Pfeifer Y, Layer F, Schaumann R, Levin K, Mielke M (2014) Inguinal skin colonization with multidrug-resistant bacteria among residents of elderly care facilities: frequency, persistence, molecular analysis and clinical impact. Int J Med Microbiol 304(8):1123-1134

4. Jans B, Schoevaerdts D, Huang TD et al (2013) Epidemiology of multidrug-resistant microorganisms among nursing home residents in Belgium. PLoS One 8:e64908

5. Gruber I, Heudorf U, Werner G et al (2013) Multidrug-resistant bacteria in geriatric clinics, nursing homes, and ambulant care - Prevalence and risk factors. Int J Med Microbiol 303(8):405-409

6. Strausbaugh LJ, Sukumar SR, Joseph CL (2003) Infectious disease outbreaks in nursing homes: an unappreciated hazard for frail elderly persons. Clin Infect Dis 36:870-876

7. Commission of the European Communities (2005) Report from the Commission to the Council on the basis of member states' reports on the implementation of the council recommendation (2002/77/ EC) on the prudent use of antimicrobial agents in human medicine. Commission of the European Comunities, Brussels

8. European Centre for Disease Prevention and Control (2014) Point prevalence survey of healthcareassociated infections and antimicrobial use in European long-term care facilities. May-September 2010. ECDC, Stockholm

9. Wischnewski N, Mielke M, Wendt C (2011) Healthcare-associated infections in long-term care facilities. German results of the European prevalence study HALT. Bundesgesundheitsblatt Gesundheitsforschung Gesundheitsschutz 54:1147-1152

10. McGeer A, Campbell B, Emori TG et al (1991) Definitions of infection for surveillance in long-term care facilities. Am J Infect Control 19:1-7

11. Stone ND, Ashraf MS, Calder J et al (2012) Surveillance definitions of infections in long-term care facilities: revisiting the McGeer criteria. Infect Control Hosp Epidemiol 33:965-977

12. Cookson B, Mackenzie D, Kafatos G et al (2013) Development and assessment of national performance indicators for infection prevention and control and antimicrobial stewardship in European long-term care facilities. J Hosp Infect 85:45-53

13. European Centre for Disease Prevention and Control (2014) Point prevalence survey of healthcareassociated infections and antimicrobial use in European long-term care facilities. April-May 2013. ECDC, Stockholm

14. Suetens C (2012) Healthcare-associated infections in European long-term care facilities: how big is the challenge? Euro Surveill 17(35)

15. Latour K, Catry B, Broex E et al (2012) Indications for antimicrobial prescribing in European nursing homes: results from a point prevalence survey. Pharmacoepidemiol Drug Saf 21:937-944
16. McClean $P$, Tunney M, Gilpin D, Parsons $C$, Hughes $C$ (2011) Antimicrobial prescribing in nursing homes in Northern Ireland: results of two point-prevalence surveys. Drugs Aging 28:819-829

17. Rummukainen ML, Karki T, Kanerva M, Haapasaari M, Ollgren J, Lyytikainen $O$ (2013) Antimicrobial prescribing in nursing homes in Finland: results of three point prevalence surveys. Infection 41:355360

18. Eilers R, Veldman-Ariesen MJ, Haenen A, Van Benthem BH (2012) Prevalence and determinants associated with healthcare-associated infections in long-term care facilities (HALT) in the Netherlands, May to June 2010. Euro Surveill 17

19. Bergman J, Schjott J, Blix HS (2011) Prevention of urinary tract infections in nursing homes: lack of evidence-based prescription? BMC Geriatr 11:69

20. Blix HS, Bergman J, Schjott J (2010) How are antibacterials used in nursing homes? Results from a point-prevalence prescription study in 44 Norwegian nursing homes. Pharmacoepidemiol Drug Saf 19:1025-1030

21. Moro ML, Ricchizzi E, Morsillo F et al (2013) Infections and antimicrobial resistance in long term care facilities: a national prevalence study. Annali di igiene: medicina preventiva e di comunita 25:109118

22. Pettersson E, Vernby A, Molstad S, Lundborg CS (2008) Infections and antibiotic prescribing in Swedish nursing homes: a cross-sectional study. Scand J Infect Dis 40:393-398

23. Fleming A, Browne J, Byrne S (2013) The effect of interventions to reduce potentially inappropriate antibiotic prescribing in long-term care facilities: a systematic review of randomised controlled trials. Drugs Aging 30:401-408

24. Furuno JP, Comer AC, Johnson JK et al (2014) Using antibiograms to improve antibiotic prescribing in skilled nursing facilities. Infect Control Hosp Epidemiol 35(Suppl 3):S56-61

25. Daneman N, Gruneir A, Bronskill SE et al (2013) Prolonged antibiotic treatment in long-term care: role of the prescriber. JAMA Intern Med 173:673682

26. Smith PW, Seip CW, Schaefer SC, Bell-Dixon C (2000) Microbiologic survey of long-term care facilities. Am J Infect Control 28:8-13

27. Gerding DN (2004) Clindamycin, cephalosporins, fluoroquinolones, and Clostridium difficile-associated diarrhea: this is an antimicrobial resistance problem. Clin Infect Dis 38:646-648

28. Owens RC Jr, Donskey CJ, Gaynes RP, Loo VG, Muto CA (2008) Antimicrobial-associated risk factors for Clostridium difficile infection. Clin Infect Dis 46 Suppl 1:S19-31

29. Chami K, Gavazzi G, Carrat F et al (2011) Burden of infections among 44,869 elderly in nursing homes: a cross-sectional cluster nationwide survey. J Hosp Infect 79:254-259

30. Smith A, Carusone SC, Loeb M (2008) Hand hygiene practices of health care workers in long-term care facilities. Am J Infect Control 36:492-494

31. Anonymous (2009) WHO guidelines on hand hygiene in health care: first global patient safety challenge clean care is safer care. Geneva
32. Boyce JM, Pittet D, Healthcare Infection Control Practices Advisory Committee, Society for Healthcare Epidemiology of America, Association for Professionals in Infection Control, Infectious Diseases Society of America, Hand Hygiene Task Force (2002) Guideline for hand hygiene in health-care settings: recommendations of the Healthcare Infection Control Practices Advisory Committee and the HICPAC/SHEA/APIC/IDSA Hand Hygiene Task Force. Infect Control Hosp Epidemiol 23:S3-40

33. Schweon SJ, Edmonds SL, Kirk J, Rowland DY, Acosta C (2013) Effectiveness of a comprehensive hand hygiene program for reduction of infection rates in a long-term care facility. Am J Infect Control 41:39-44

34. Nationales Referenzzentrum für Surveillance von nosokomialen Infektionen (2014) KISS Krankenhaus-Infektions-Surveillance-System. Modul HAND-KISS_P Referenzdaten, Berechnungszeitraum:1. Januar 2013 bis 31. Dezember 2013. NRZ, Berlin

35. Nationales Referenzzentrum für Surveillance von nosokomialen Infektionen (2014) Aktion saubere Hände. http://www.aktion-sauberehaende.de/ ash/. Zugegriffen: 01. Okt 2014

36. Rummukainen $M$, Jakobsson $A$, Karppi $P$, Kautiainen $\mathrm{H}$, Lyytikainen $\mathrm{O}$ (2009) Promoting hand hygiene and prudent use of antimicrobials in longterm care facilities. Am J Infect Control 37:168-171

37. Comondore VR, Devereaux PJ, Zhou Q et al (2009) Quality of care in for-profit and not-for-profit nursing homes: systematic review and meta-analysis. BMJ 339:b2732 